\title{
Towards comprehensive early abortion service delivery in high income countries: insights for improving universal access to abortion in Australia
}

\author{
Angela Dawson ${ }^{1 *}$ (D) Deborah Bateson ${ }^{2}$, Jane Estoesta $^{3}$ and Elizabeth Sullivan ${ }^{4}$
}

\begin{abstract}
Background: Improving access to safe abortion is an essential strategy in the provision of universal access to reproductive health care. Australians are largely supportive of the provision of abortion and its decriminalization. However, the lack of data and the complex legal and service delivery situation impacts upon access for women seeking an early termination of pregnancy. There are no systematic reviews from a health services perspective to help direct health planners and policy makers to improve access comprehensive medical and early surgical abortion in high income countries. This review therefore aims to identify quality studies of abortion services to provide insight into how access to services can be improved in Australia.
\end{abstract}

Methods: We undertook a structured search of six bibliographic databases and hand-searching to ascertain peer reviewed primary research in English between 2005 and 2015. Qualitative and quantitative study designs were deemed suitable for inclusion. A deductive content analysis methodology was employed to analyse selected manuscripts based upon a framework we developed to examine access to early abortion services.

Results: This review identified the dimensions of access to surgical and medical abortion at clinic or hospitaloutpatient based abortion services, as well as new service delivery approaches utilising a remote telemedicine approach. A range of factors, mostly from studies in the United Kingdom and United States of America were found to facilitate improved access to abortion, in particular, flexible service delivery approaches that provide women with cost effective options and technology based services. Standards, recommendations and targets were also identified that provided services and providers with guidance regarding the quality of abortion care.

Conclusions: Key insights for service delivery in Australia include the: establishment of standards, provision of choice of procedure, improved provider education and training and the expansion of telemedicine for medical abortion. However, to implement such directives leadership is required from Australian medical, nursing, midwifery and pharmacy practitioners, academic faculties and their associated professional associations. In addition, political will is needed to nationally decriminalise abortion and ensure dedicated public provision that is based on comprehensive models tailored for all populations.

\footnotetext{
* Correspondence: angela.dawson@uts.edu.au

${ }^{1}$ Faculty of Health, University of Technology, Sydney (UTS), P.O. Box 123,

Ultimo, NSW 2007, Sydney, NSW, Australia

Full list of author information is available at the end of the article
} 


\section{Background}

Access to a safe, early induced abortion, or the termination of an unplanned pregnancy (up to and including 13 weeks [1] can potentially prevent the death of nearly half a million women and the associated morbidity of another five million women worldwide [2]. Improving access to safe abortion is an essential strategy in the provision of universal access to reproductive health and achieving the sustainable development goals [3].

Comprehensive safe abortion care encompasses the provision of elective abortion services at the request of the woman, along with counselling for contraceptive use, medical after-care, and attention to other issues that are relevant to the woman's health [4]. The provision of safe medical and/or surgical abortion requires an enabling legal, regulatory and policy environment that is responsive to women's needs and community demands for quality care, services and information. The legal grounds upon which a woman is permitted to have an abortion differ across and within countries. However, a woman's access can also be affected by the: availability and quality of health services, geographical access to services, financial affordability and socio-cultural acceptability of the procedure/s and associated care.

Planning, delivering and evaluating legal and safe, comprehensive abortion services that enable women to make a choice about the use of such services requires attention to a number of principles that were developed by the World Health Organization (WHO) through a high level consultative process. These quality service delivery principles include the establishment of national standards and guidelines, integrated multidisciplinary services, ensuring health-care provider skills and performance, the costing and financing of services, and a systematic approach to policy and programme development.

Despite numerous guidelines and recommendations there has been little research from a health services approach to help direct health planners and policy makers to increase access to abortion. Souza et al. have noted gaps in best practice with regard to integrating abortion services into existing family planning services and optimizing the workforce [5]. However, although task shifting from doctors to nurses and midwives in high income countries (HIC) (countries with a gross national income per capita of $\$ 12,476$ or greater) [6] shows promise [7], there is a lack of evidence in this area [8]. The few systematic reviews that are available in the area of service delivery include an examination of the acceptability of home and clinic based medical abortion by Ngo et al. [9] in largely low and middle income countries (LMIC) with the exception of one study from France. Another review comprising three studies from Italy, Scotland and Iceland found no evidence of increased acceptance and use of contraceptive methods after an abortion [10]. The review by Doren at al. [11] examines factors that facilitate or constrain access to abortion from the perspective of women and providers. Despite these studies there are no systematic reviews that provide insight into models of comprehensive medical and surgical abortion services. This review therefore aims to identify quality studies of abortion services to provide insight into how access to services can be improved in Australia.

\section{The Australian context}

There is no national data collection on the incidence of induced abortion in Australia. Notification of abortions is mandatory in the States of South Australia, Western Australia and the Norther Territory but only South Australia and Western Australia publish data on induced abortions [12, 13]. Several studies have estimated the number of abortions in Australia of over 80,000. The estimated number of induced abortions adjusted for patients who do not claim Medicare (National Reimbursement Services) were 84218 and 83210 in 2013 and 2014, respectively. The national age-standardised abortion rate was 19.7 per 1000 women in 2003 and 19.3 per 1000 women in 2004. However, the latest information was from 2004 [14-16].

The situation in Australia is further complicated with marked differences in abortion legislation between states and territories. Of the nine laws that govern abortion in the country, restrictions exist in all with the exception of the Australian Capital Territory. This highlights the urgent need for reform [17]. The recent focus at the national level has been on new Medicare Benefit payments for pregnancy support counselling that has been poorly accessed by women with unplanned pregnancies [18].

The Australian public including health professionals are largely supportive of the provision of abortion $[19,20]$ and its decriminalization [21]. However, the lack of data and the complex legal and service delivery situation impacts upon access for women seeking an early termination of pregnancy. Recent literature cites issues related to geographical and financial access $[22,23]$ and considerable gaps in women's knowledge [24]. Services are provided across a myriad of public and private contexts where there is no accepted and standardized approach for service delivery that facilitates universal access to comprehensive abortion care. This paper therefore aims to identify service delivery models of comprehensive abortion care, both medical and surgical, that has been effective in increasing access to early abortion care in high income country settings. Such insights may provide Australia with practical evidence-based policy options for improving access to abortion services. 


\section{A framework for understanding the factors contributing to universal access to comprehensive abortion services} Access can be understood from supply and demand side perspectives [25]. Demand for a service is influenced by factors that determine whether a woman is willing and able to seek appropriate abortion care. Supply is determined by factors that include availability, technology, management, and price that interact to produce effective health care. These perspectives can be considered under various dimensions of access that are well described in the literature [26, 27]. We have summarised these dimensions in a framework adapted from existing literature $[28,29]$ (see Table 1) in order to conceptualise questions that can be used to examine the extent of access to abortion services.

\section{Methods}

Six bibliographic databases (Medline, PubMed, Web of Science, ProQuest Health and Medicine, Scopus, Science Direct), Google Scholar and the reference lists of identified papers were methodically searched to retrieve research literature. A deductive qualitative content analysis methodology was employed to analyse selected primary research manuscripts [30].

A Population, Interventions, Comparators, Outcomes, Study design question guided the development of the review question [31]. The question we sought to answer was: for services providing first trimester abortion in high income country settings, what approaches to service delivery have been found to be effective in increasing access? We aimed to include studies with demonstrable outcomes from both supply and demand perspectives while acknowledging important health systems factors that enable service delivery. We sought studies examining comprehensive abortion services, as well those investigating components of abortion services with a focus on understanding new or existing delivery systems and key processes as defined by Donadedian [32]. Outcomes of interests include the abortion service delivered, changes in behaviour such as contraception uptake, change in health status such as complete terminations and women's access to abortions services as defined in Table 1 including satisfaction and uptake of services.

We defined comprehensive abortion service delivery as the provision of legal, safe, stigma free, high quality services that include abortion, post abortion care, contraception and referral. This also involves attention to issues concerning information provision, initial assessment and arrangements for the procedure as outlined in the recent best practice paper by the Royal College of Obstetricians and Gynaecologists (RCOG) [33] and service factors identified by IPAS [34].

Table 1 A framework for examining access to early abortion services

\begin{tabular}{ll}
\hline Access Dimensions & $\begin{array}{l}\text { Questions to establish extent of access to comprehensive abortion services-what is } \\
\text { done and how }\end{array}$
\end{tabular}

Availability: The number of existing abortion services meet women's needs

How many and what types of abortion services exist? Which organizations offer these services? Are there enough willing and skilled personnel to deliver abortion services? Do the services offer choices that correspond with the needs of financially disadvantaged women? Are the services and supplies of abortion drugs and associated equipment and tests sufficient to cover the demand?

Accessibility: Abortion services are near where women live, or accessible in their homes and referral timely

Affordability: The prices of terminations are aligned with women's income and ability to pay

\section{Adequacy: The organization of abortion services meet women's expectations}

Acceptability: The services are satisfactory and used by women

Quality: The services are scientifically and medically appropriate and of good quality
What is the geographical distance between abortion services and the homes of women? Can services be reached and women referred in a timely and stress-free manner? How has technology been employed to address issues of distance?

What are the direct costs of abortion services and the associated commodities? What are the indirect costs in terms of transportation, childcare, lost time and income? Are payment options available for women? What are the costs to the health system and are they affordable?

How are/is the abortion service/s organized? Does the organizational structure meet women's expectations? Do opening hours match the schedules of women? Are facilities clean, organized and well managed?

Does the information, explanation, and service provided accommodate social norms and values? Do women find self-management aspects such as drug taking and pregnancy testing satisfactory and easy to use? Do women feel welcome and cared for? Do women trust in the competence and character of the health care providers?

Are there service delivery standards and quality management systems? How are women's complaints dealt with? What quality assurance, control and improvement mechanisms are in place? Quality also extends to the manner in which women are treated, and how underlying determinants of health are addressed. Is there provider sensitivity training (i.e. in appropriate language use, cultural safety, privacy) or/and incentive schemes for providers to offer services? Are providers accredited, and regulated? Do providers collect accurate data? Is family planning/contraceptive advice provided? How are abortifacient drugs packaged? Is treatment observed if required? What family planning training and peer support is available for providers? 
In line with other systematic reviews in the field $[35,36]$ a structured search of contemporary research literature was conducted between 2005 and 2015 using key words: "abortion" OR "termination of pregnancy" AND "service delivery" OR "model of care". We sought to assess the results of peer reviewed primary research literature therefore grey resources were excluded. Retrieved records were first screened for their focus on abortion service delivery according to the review question by the first author and duplicates removed. According to the inclusion/exclusion criteria (see Table 2) studies whose focus was outside of the aim, along with discursive papers and those older than 10 years were removed. The Preferred Reporting Items for Systematic Reviews and Meta-Analyses (PRISMA) guidelines were employed to report the review process [37] (see Fig. 1). The sources and numbers of papers retrieved and screened according to their relevance are outlined at Table 3. Most papers were then excluded (999) at closer examination as they were not concerned with specific service delivery aspects the paper by Loeber is one example [38].

Forty-one papers were appraised using the Critical Appraisal Skills Programme tool for qualitative research [39] and Pluye et al.'s [40] scoring system was used to assess the non-experimental and mixed method studies. Fourteen items were discarded due to methodological concerns. The lead author first appraised the papers using the checklists of both tools which were independently verified by the DB and ES.

Data were extracted from the twenty-nine papers and first described according to characteristics of the research (e.g., primary author, year of publication, study setting); research participants (age, gender, and socio-demographic data); study aim; study design, and findings (see Table 3). The conceptual framework outlined at Table 1 was then applied to identify the dimensions of access and demand or supply perspective. A content analysis of the extracted text relating to each dimensions of access was performed by the first author and then discussed with all authors. The questions aligned with each dimension of access as were used to interrogate the findings of the paper. We then identified evidence of processes and outcomes that contributed to the ability of the service to increase access to abortion, as well as the constraints, to provide insight into how access to services could be improved in Australia.

\section{Results}

Twenty-nine papers were included in this review (see Table 3). Fourteen papers were from the United Kingdom (UK) [41-54], seven from the United States (US) [55-61], two from Russia [62, 63], two from Australia [22, 23], three from New Zealand (NZ) [64-66] and one Canadian paper [67]. Of these papers all were quantitative except for three qualitative studies [22, 57, 61].

We categorized the papers according to the aspect of the abortion service under study, outcome measures, dimensions of access covered (see Table 4) and their focus on supply, demand or both (see Table 5). Of these, six papers focus specifically on service delivery related to aspects of surgical termination of pregnancy (STOP) while nine papers were concerned with medical termination (MTOP). Other papers describe both types of abortion with service related outcomes according to the components of care [48, 63], approach to service integration [45], referral, booking and counselling [43, 44, 49, 56, 67] and the type of facility where the services are provided $[23,46,60,61,64-66]$. Of the access dimensions covered, least covered were adequacy and affordability with supply focused papers gaining more attention than those solely concerned with demand side issues. The results of the analysis of the findings section of the 29 papers as per the questions generated from the dimensions in Table 1 are described below. We have specifically reported factors that have been identified to enhance access service delivery and those that have been found to constrain access to services.

Table 2 The inclusion/exclusion criteria applied to the screening of papers for the review

\begin{tabular}{|c|c|}
\hline Included & Excluded \\
\hline $\begin{array}{l}\text { Health service research examining how women access health providers } \\
\text { and early abortion services, how much care costs, and what happens to } \\
\text { women as a result of this care }\end{array}$ & $\begin{array}{l}\text { Clinical studies examining safety and effectiveness of medications, devices } \\
\text { diagnostic products and treatment regimens intended for early abortion }\end{array}$ \\
\hline $\begin{array}{l}\text { Research examining first trimester abortion service delivery processes, } \\
\text { activities, strategies or components with the goal of improving } \\
\text { provision, quality, utilization, coverage, efficiency, and equity }\end{array}$ & $\begin{array}{l}\text { Research examining input aspects or resources of abortion care such as } \\
\text { human resources, insurance schemes, drug supply or procurement, } \\
\text { national policy or governance }\end{array}$ \\
\hline Primary research paper & Discursive or descriptive outlines of projects, conference abstracts \\
\hline High income country & Upper middle, middle, lower middle and low income country setting \\
\hline English & Non English \\
\hline$>2005$ & $<2005$ \\
\hline
\end{tabular}




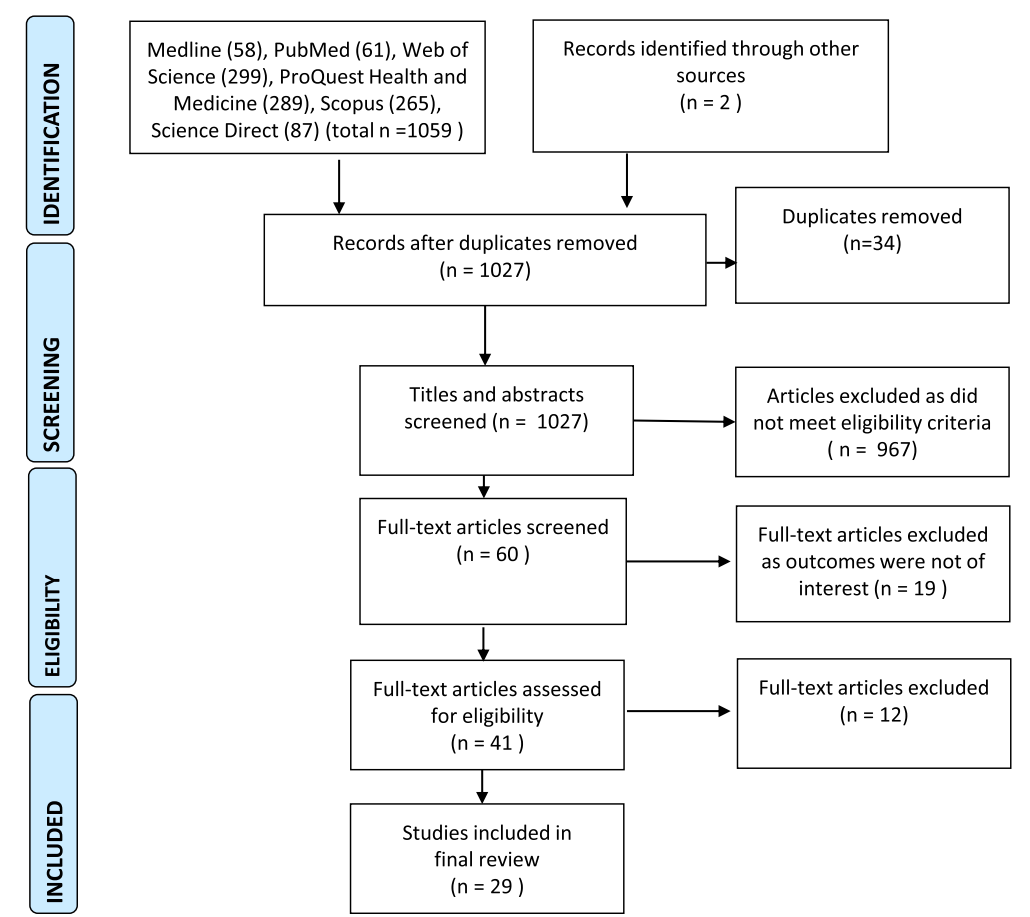

Fig. 1 The Literature review selection process. Preferred Reporting diagram for Systematic Reviews and Meta-analyses (PRISMA) showing selection of publications for review

\section{Availability}

The availability of both MTOP and STOP services at facilities was found in one study to increase access. Tupper et al. describe the development of a new MTOP service in the UK to address a gap in the public sector provision of abortion; and to increase the timeliness and choice of type of first trimester abortion for women in an area where only STOP had previously been available and MTOP via an out of area private provider [53]. This retrospective audit of the first 12 months of the service indicate that it went some way towards addressing the need for an increase in the availably of MTOP and meeting the Department of Health targets on TOP to reduce waiting times and increase the number of women provided with an abortion under 10 weeks' gestation. The findings show that all available appointments were filled in the first week that the service was opened however some patients had to be referred in the first 8 months due to a lack of staffing capacity. The service was therefore extended to accommodate the larger than anticipated demand resulting in lower numbers of onward referrals. Another UK study evaluated a newly established, public and NGO sector MTOP service to increase women's choice in an area where STOP was only available. However the impact of the new service was not reported other than the numbers of successful early MTOP procedures [46].
Three papers in the review outline the NGO, Planned Parenthood's efforts to increase the availability of MTOP in the one State in the US by providing medical abortion via telemedicine at clinics without an on-site physician [57-59]. Women noted the service enabled them to undergo the procedure sooner without waiting for an appointment which ensured the availability of MTOP as per the gestational age limit. Staff noted that the service was able to achieve a greater reach and offer more choice for abortion, as well as access to timely same day treatment. Staff stated that they found the process of introducing the service useful in building their skills and ability to provide new services [57]. There was an increase in the proportion of medical abortions in the clinics from 46 to $54 \%$. Clinic patients had increased odds of obtaining both MTOP before 13 weeks' gestation after the introduction of telemedicine $[58,59]$.

Another paper examined the use of a telephone booking service to increase the availability abortion via manual vacuum aspiration (MVA) under local anaesthesia in a local English hospital [52]. The study established that demand from referrers for the telephone booking clinic was greater than could be accommodated by the service [52]. However, substituting two doctor assessment clinics of 10 patients for two clinics of six patients, supported by 2.5 nurse telephone clinics, was able to increase the service capacity by $25 \%$. 


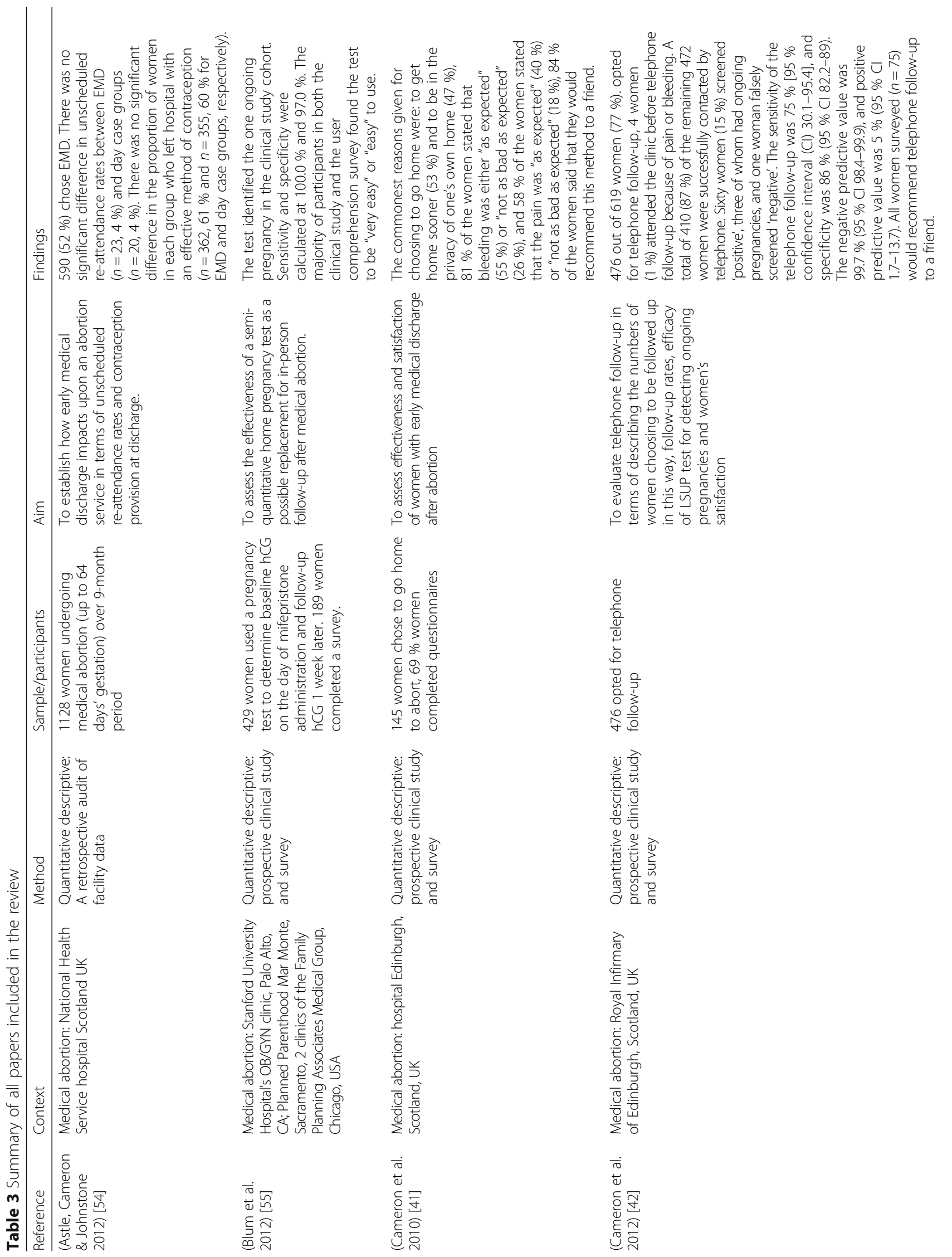




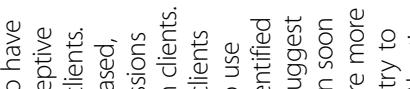

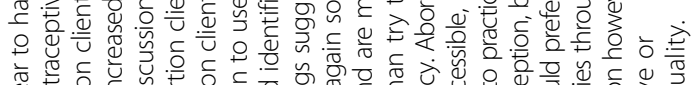

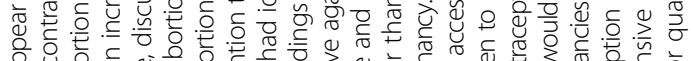

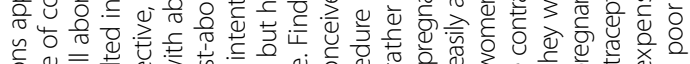

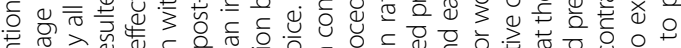

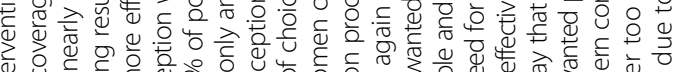

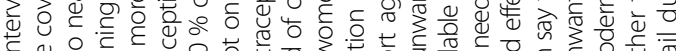

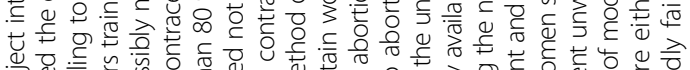

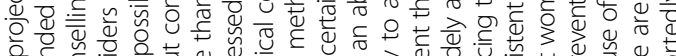

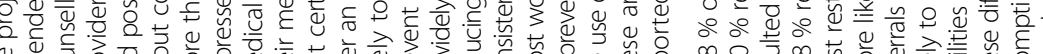

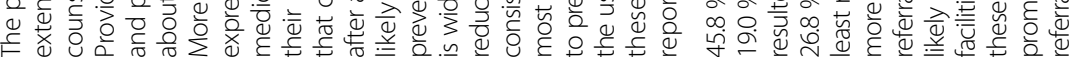

离

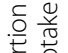

일

落觉

o.

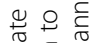

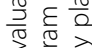

ঐ) 흘

8;

总

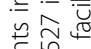

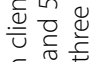

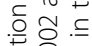

每

要.

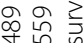

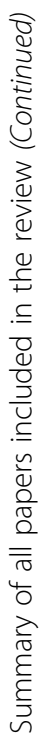

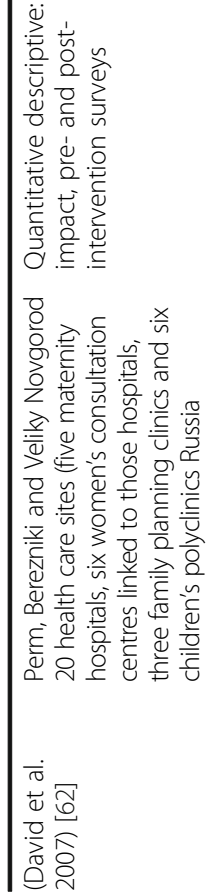

东

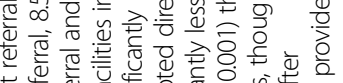

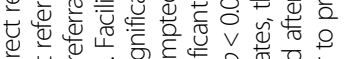

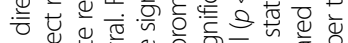

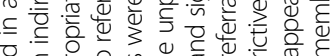

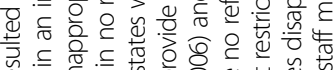

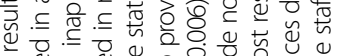

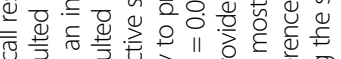

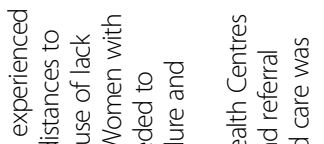

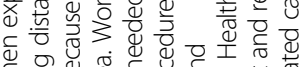

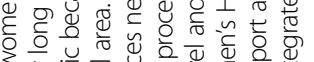

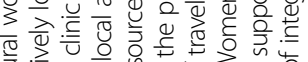

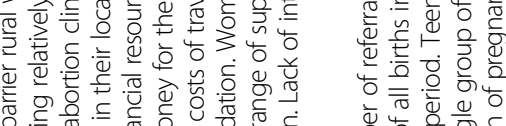

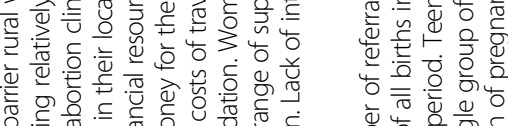
늘

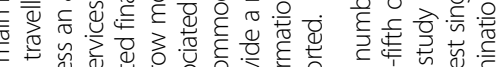

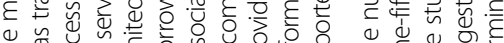

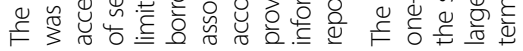
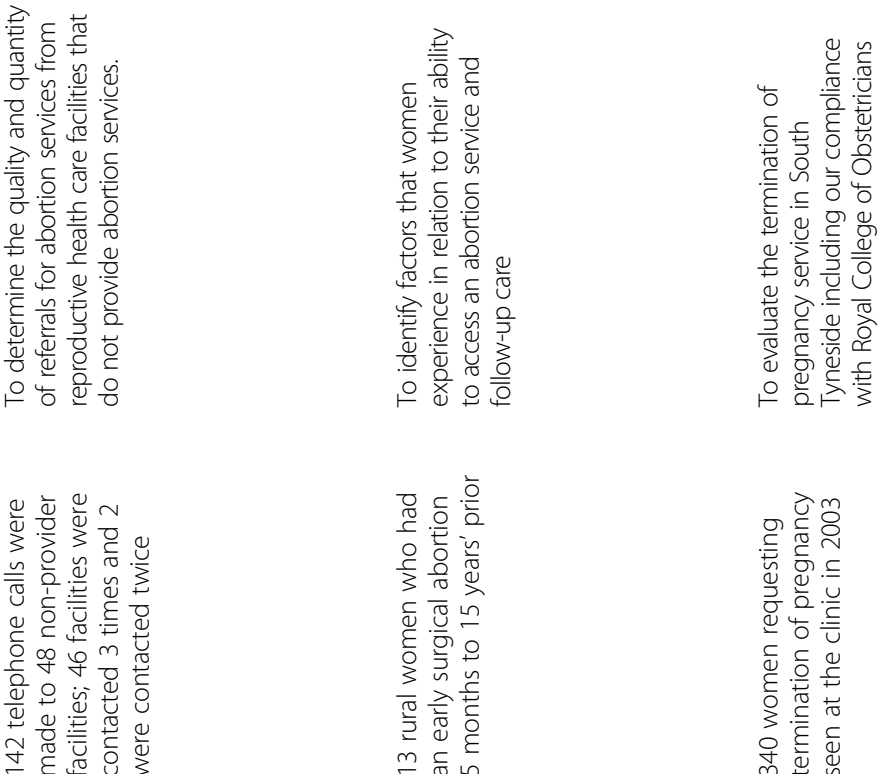

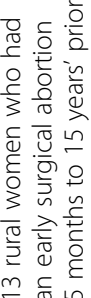

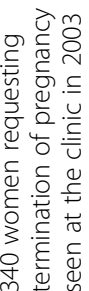

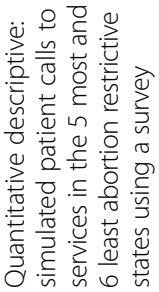

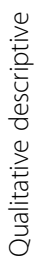

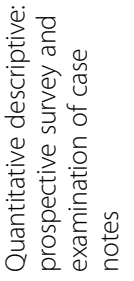

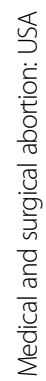
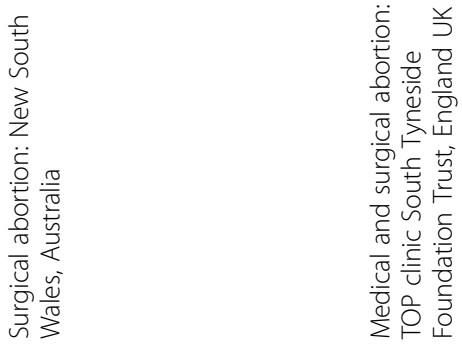

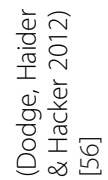

$\overline{8}$

공

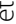

㞻完 


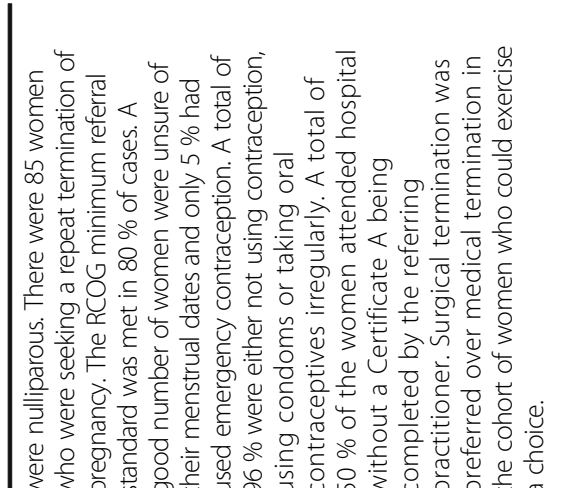

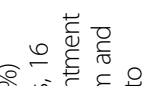

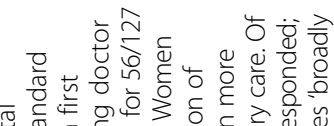

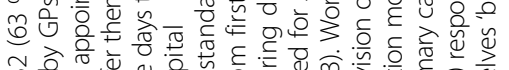

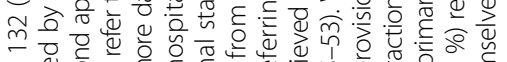

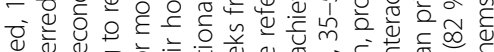

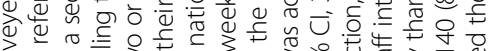

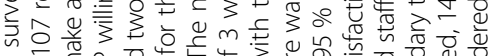

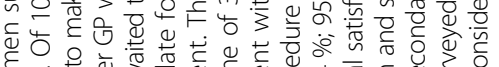

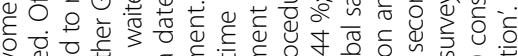

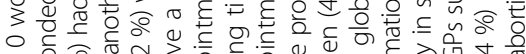

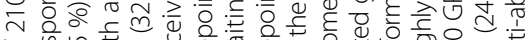

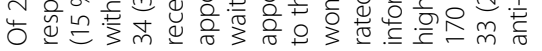
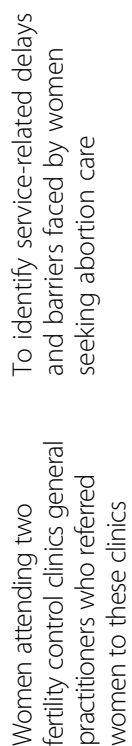

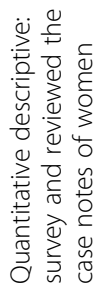

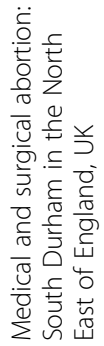

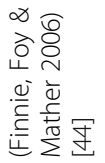
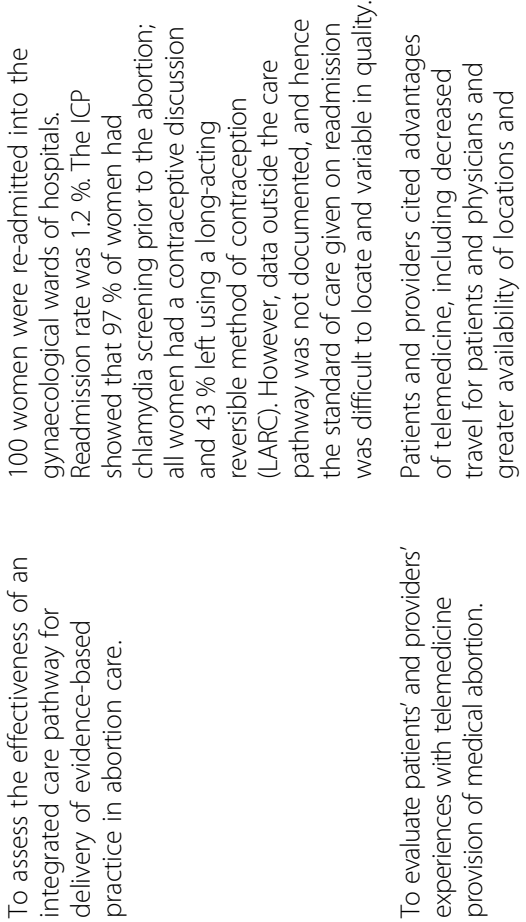

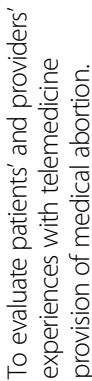
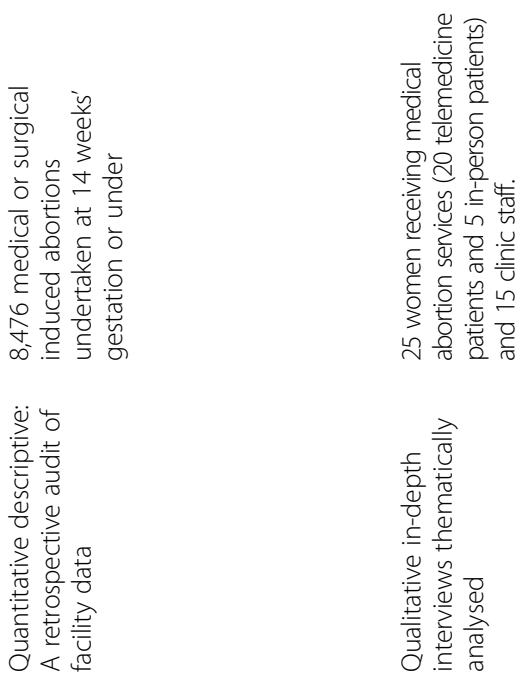

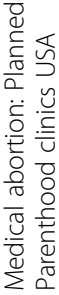

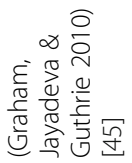

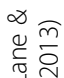

离产 這愌会 


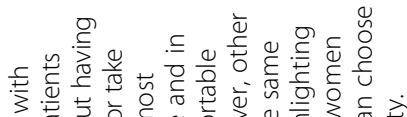

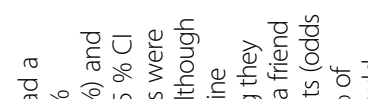

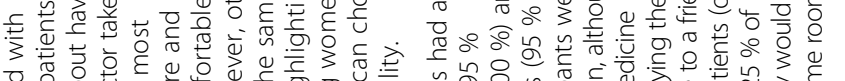

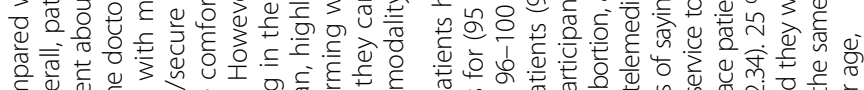

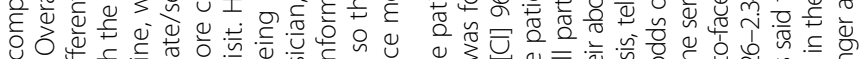

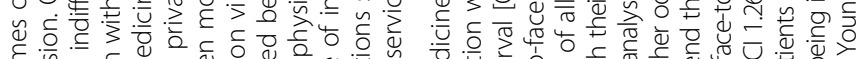

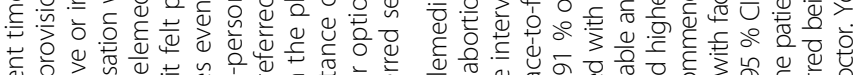

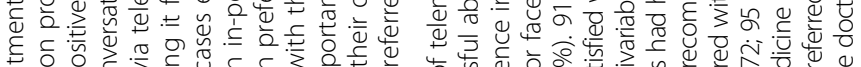

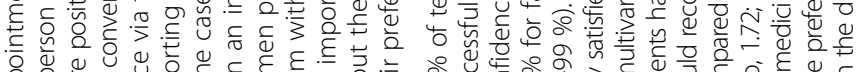

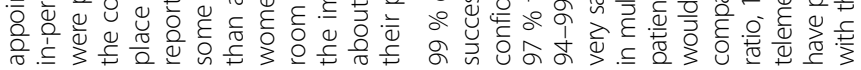

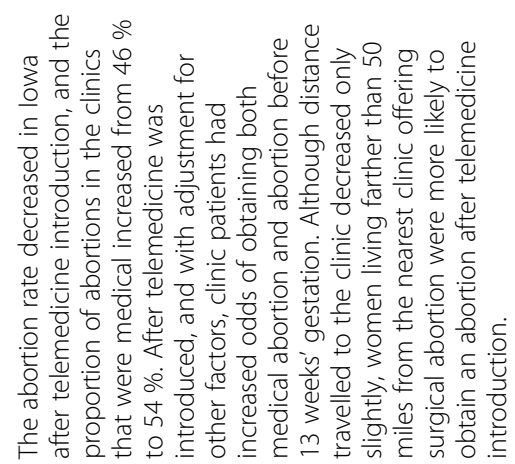

¿

苛

을 $\frac{\stackrel{d}{\varrho}}{\frac{0}{\ell}}$

范

ரृ

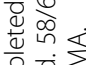

है एं

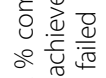

๙ $\frac{\pi}{3} \frac{n}{3}$
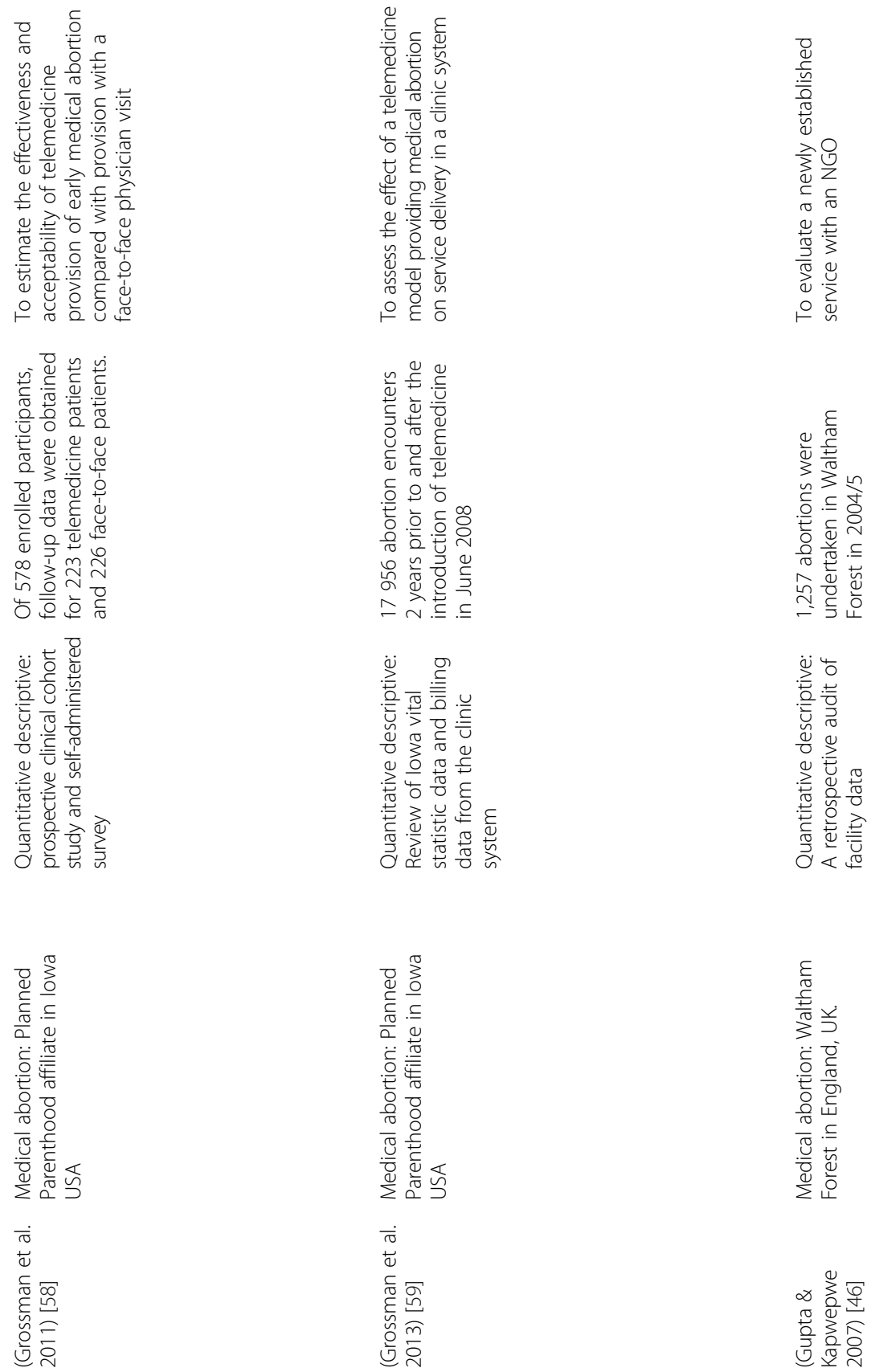

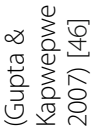




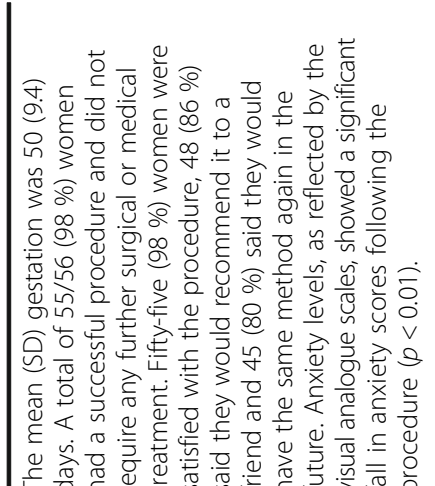
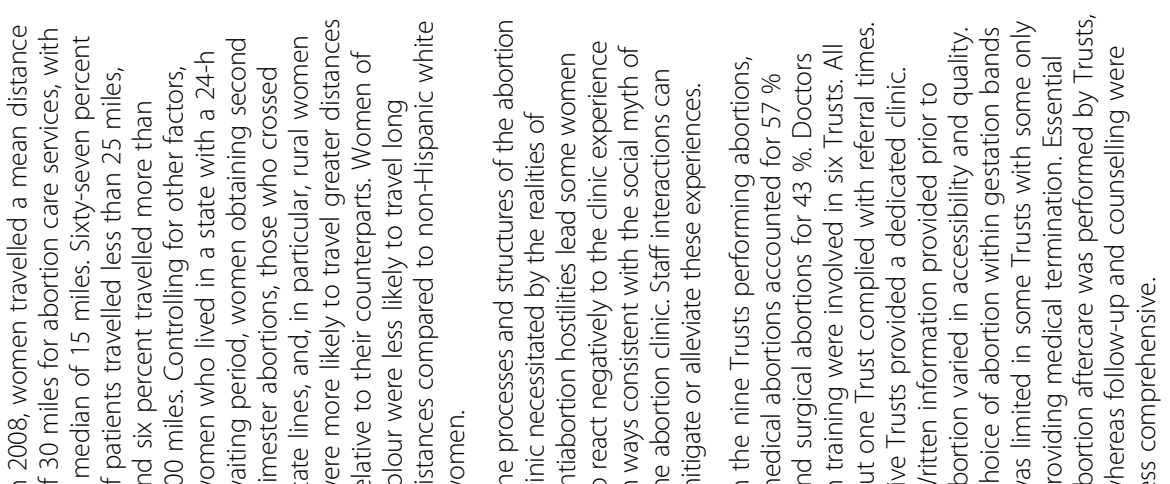

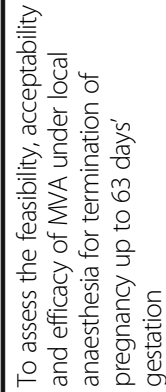

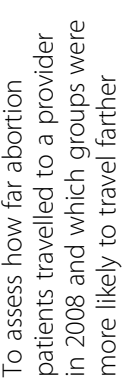

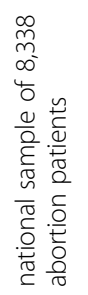

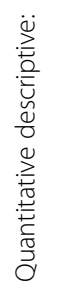

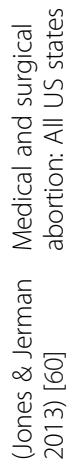

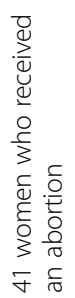

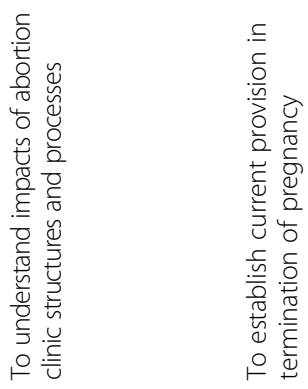

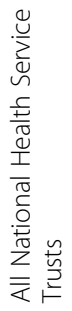

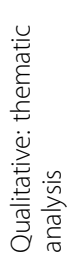

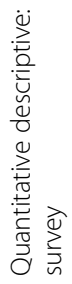

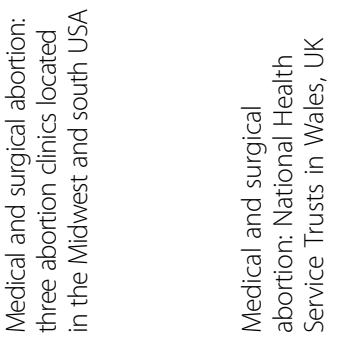

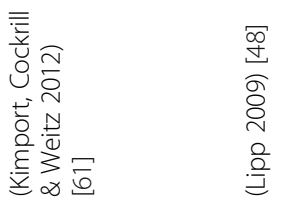



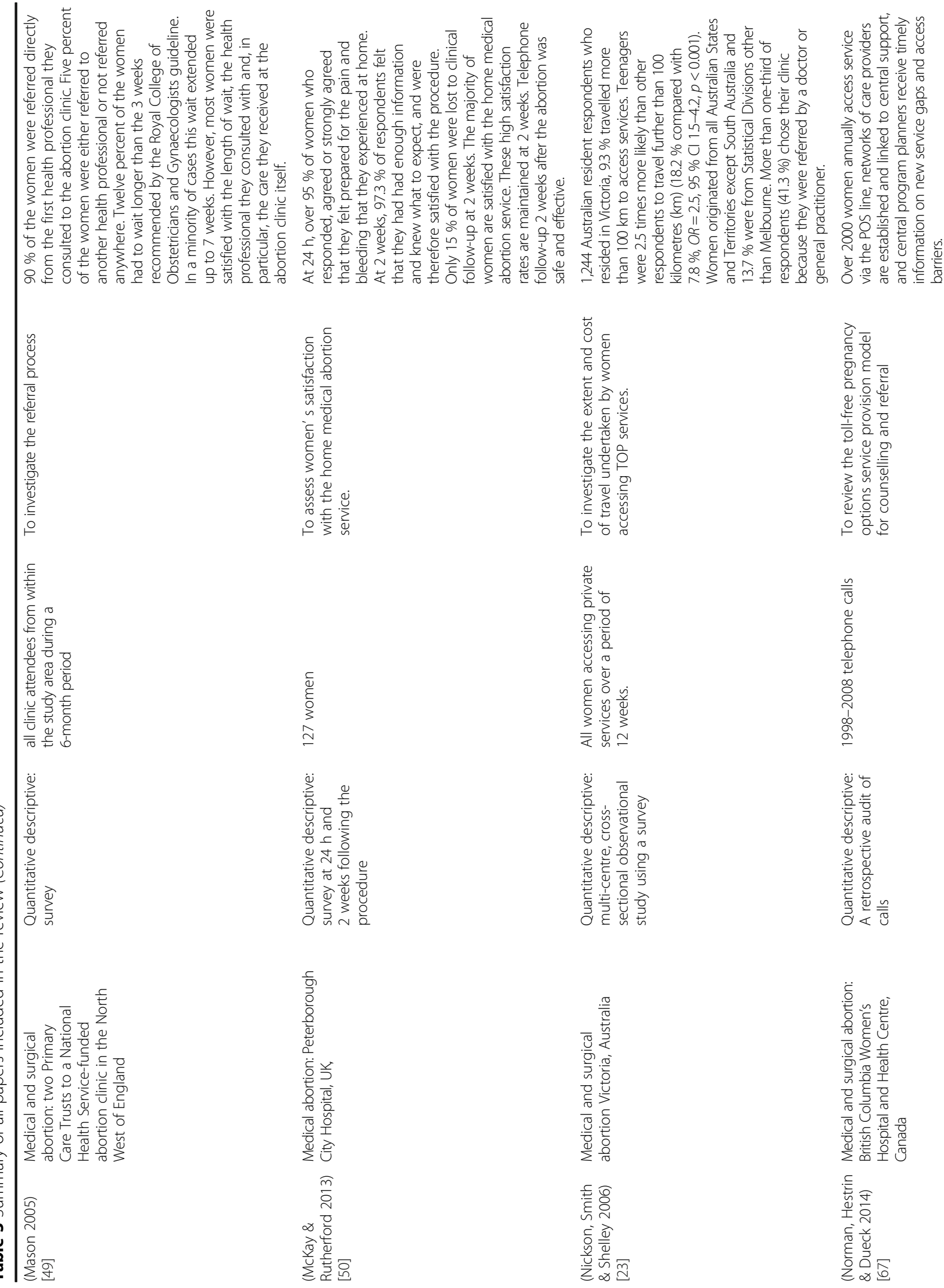


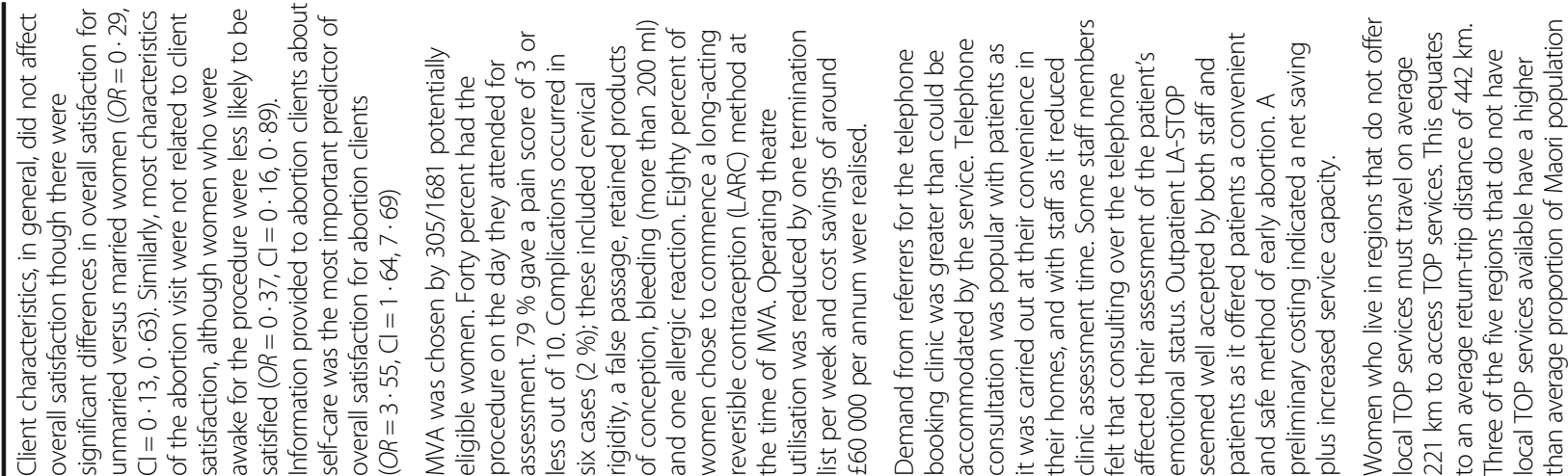
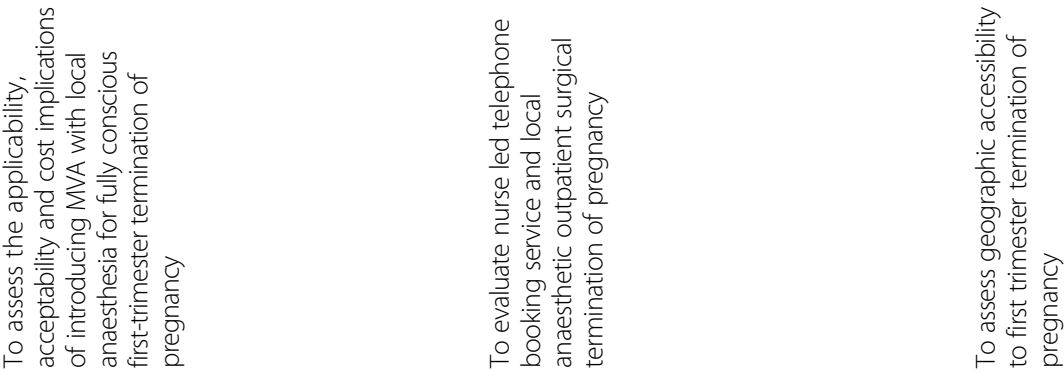

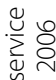

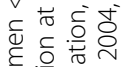

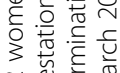

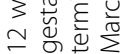

$\stackrel{\circ}{\circ} \stackrel{0}{\underline{t}}$

$\frac{1}{ㅇ ㅡ ㅇ ㅡ ㄴ ~}$

3

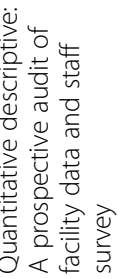

要需要

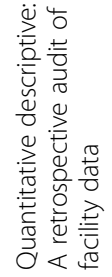

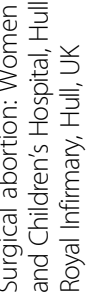

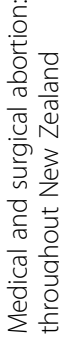




\begin{tabular}{|c|c|c|}
\hline 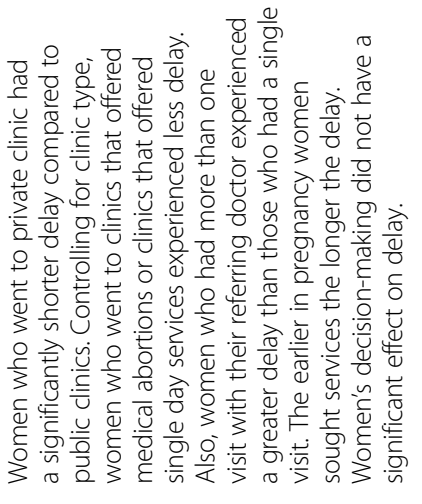 & 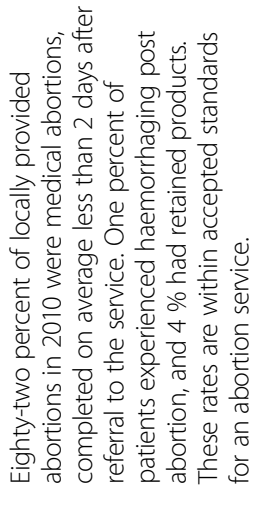 & 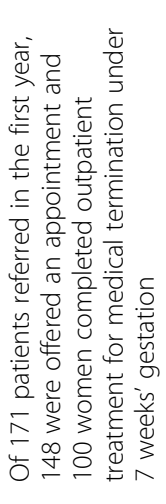 \\
\hline 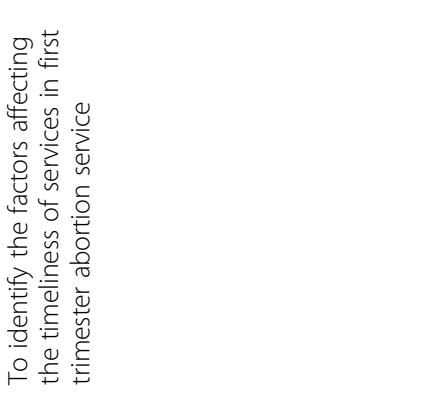 & 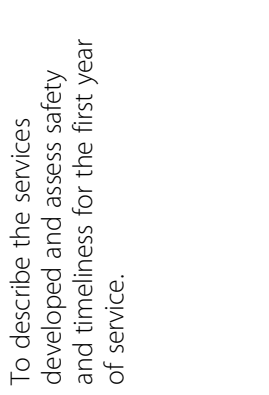 & 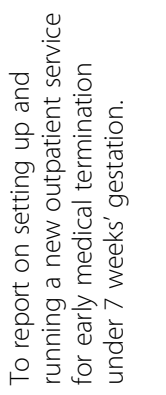 \\
\hline 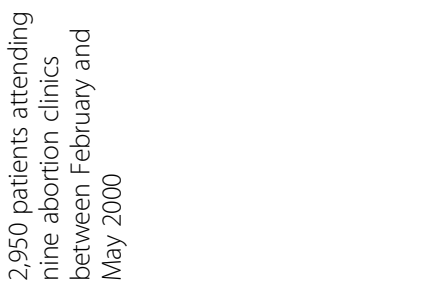 & 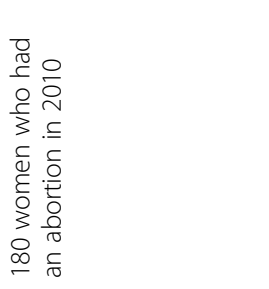 & 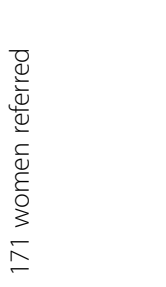 \\
\hline 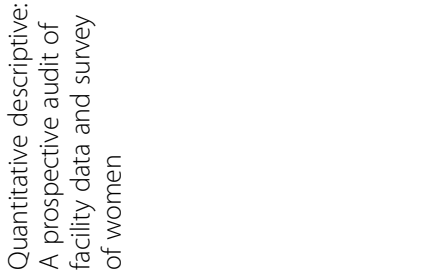 & 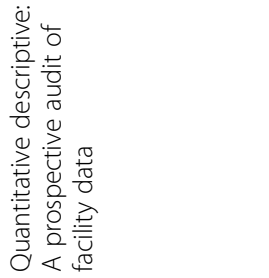 & 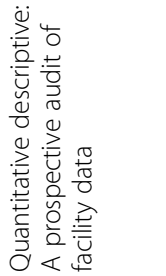 \\
\hline 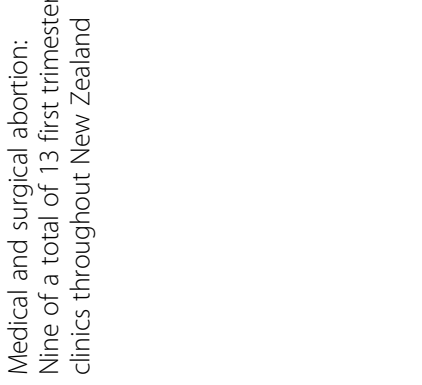 & 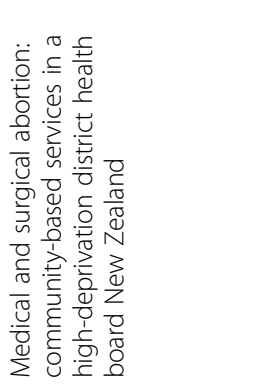 & 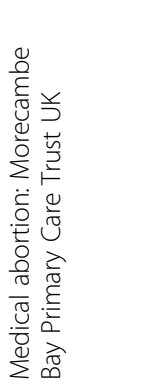 \\
\hline 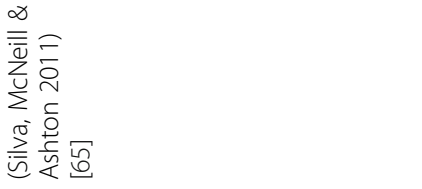 & 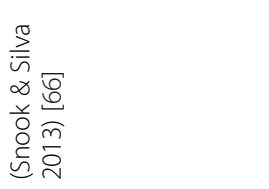 & 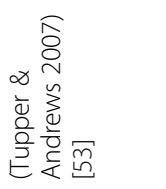 \\
\hline
\end{tabular}


Table 4 Access Dimensions Covered across papers in the review

\begin{tabular}{|c|c|c|c|c|c|c|}
\hline & Availability & Accessibility & Affordability & Adequacy & Acceptability & Quality \\
\hline (Astle, Cameron \& Johnstone 2012) [54] & & & & & & $\sqrt{ }$ \\
\hline (Blum et al. 2012) [55] & & & & & $\sqrt{ }$ & \\
\hline (Cameron et al. 2010) [41] & & & & & $\sqrt{ }$ & $\sqrt{ }$ \\
\hline (Cameron et al. 2012) [42] & & & & & $\sqrt{ }$ & \\
\hline (David et al. 2007) [62] & $\sqrt{ }$ & & & & & $\sqrt{ }$ \\
\hline (Dodge, Haider \& Hacker 2012) [56] & & $\sqrt{ }$ & & & & \\
\hline (Doran \& Hornibrook 2014) [22] & $\sqrt{ }$ & $\sqrt{ }$ & $\sqrt{ }$ & & $\sqrt{ }$ & \\
\hline (Esen et al. 2006) [43] & & $\sqrt{ }$ & & & & $\sqrt{ }$ \\
\hline (Finnie, Foy \& Mather 2006) [44] & & $\sqrt{ }$ & & & $\sqrt{ }$ & $\sqrt{ }$ \\
\hline (Graham, Jayadeva \& Guthrie 2010) [45] & & & & & & $\sqrt{ }$ \\
\hline (Grindlay, Lane \& Grossman 2013) [57] & $\sqrt{ }$ & $\sqrt{ }$ & $\sqrt{ }$ & $\sqrt{ }$ & $\sqrt{ }$ & \\
\hline (Grossman et al. 2011) [58] & & & & & $\sqrt{ }$ & $\sqrt{ }$ \\
\hline (Grossman et al. 2013) [59] & $\sqrt{ }$ & $\sqrt{ }$ & & & & \\
\hline (Gupta \& Kapwepwe 2007) [46] & $\sqrt{ }$ & & & & & $\sqrt{ }$ \\
\hline (Hamoda et al. 2005) [47] & & & & & $\sqrt{ }$ & $\sqrt{ }$ \\
\hline (Jones \& Jerman 2013) [60] & & $\sqrt{ }$ & & & & \\
\hline (Kimport, Cockrill \& Weitz 2012) [61] & & $\sqrt{ }$ & & $\sqrt{ }$ & $\sqrt{ }$ & \\
\hline (Lipp 2009) [48] & $\sqrt{ }$ & & & & & $\sqrt{ }$ \\
\hline (Mason 2005) [49] & & $\sqrt{ }$ & & & & \\
\hline (McKay \& Rutherford 2013) [50] & & & & & $\sqrt{ }$ & \\
\hline (Nickson, Smith \& Shelley 2006) [23] & & $\sqrt{ }$ & $\sqrt{ }$ & & $\sqrt{ }$ & \\
\hline (Norman, Hestrin \& Dueck 2014) [67] & $\sqrt{ }$ & & & & $\sqrt{ }$ & $\sqrt{ }$ \\
\hline (Oliveras, Larsen \& David 2005) [63] & & & & $\sqrt{ }$ & $\sqrt{ }$ & \\
\hline (Pillai et al. 2015) [51] & & & $\sqrt{ }$ & & $\sqrt{ }$ & \\
\hline (Sharma \& Guthrie 2006) [52] & $\sqrt{ }$ & & $\sqrt{ }$ & & $\sqrt{ }$ & \\
\hline (Silva \& McNeill 2008) [64] & & $\sqrt{ }$ & & & & \\
\hline (Silva, McNeill \& Ashton 2011) [65] & & $\sqrt{ }$ & & & & \\
\hline (Snook \& Silva 2013) [66] & $\sqrt{ }$ & $\sqrt{ }$ & & & & $\sqrt{ }$ \\
\hline (Tupper \& Andrews 2007) [53] & $\sqrt{ }$ & & $\sqrt{ }$ & & & $\sqrt{ }$ \\
\hline
\end{tabular}

Other studies in the review identified the impact of the health workforce on the availability of abortion services. A shortage of doctors, particularly female doctors who were willing to perform abortions was noted by staff of women's health centres in rural Australia. In addition, staff cited a shortage of rural general practitioners as impacting upon the availability of referral points and follow up care [22]. A study of GPs $(\mathrm{No}=126)$ in the north east of England UK found that approximately onethird surveyed did not provide on-site pregnancy testing (52; $37 \%$ ) and believed they had insufficient information about abortion services (43; $32 \%$ ) [44]. It is suggested that these factors affected service availability.

One study from NZ showed that a newly established community-based abortion clinic in one district health board (DHB) developed to provide local service provision and prevent out-of-region referrals was able to contribute to addressing the need for increased availability. Of the 180 women from the DHB who had an abortion in 2010, $81 \%(n=145)$ had an abortion locally, while the remaining $19 \%$ went outside the region for the service [66]. However, service availability did not appear to be equitable as three of the five regions that did not have abortion services were found to have a higher than average proportion of a largely disadvantaged Indigenous Maori population.

\section{Accessibility}

Two studies in the review investigated the use of technology to address accessibility issues, in particular to eliminate travelling time and overcome geographical barriers to abortion services. Qualitative interviews with women who selected a MTOP telemedicine clinic stated that they did so due to logistical concerns as they were unable to access 
Table 5 Approach to abortion service, outcomes, access dimension and perspective

\begin{tabular}{|c|c|c|c|}
\hline & Aspect of abortion service under study & Outcome measures & $\begin{array}{l}\text { Supply and/or demand } \\
\text { perspective }\end{array}$ \\
\hline & $\begin{array}{l}\text { Outpatient/home based/early medical } \\
\text { discharge (MTOP) }\end{array}$ & & \\
\hline $\begin{array}{l}\text { (Astle, Cameron \& Johnstone } \\
\text { 2012) [54] }\end{array}$ & & $\begin{array}{l}\text { Unscheduled re-attendance rates, contraception } \\
\text { provision at discharge rate }\end{array}$ & Supply \\
\hline (Cameron et al. 2010) [41] & & Women's satisfaction & Demand \\
\hline $\begin{array}{l}\text { (McKay \& Rutherford } \\
\text { 2013) [50] }\end{array}$ & & Women's satisfaction & Demand \\
\hline (Tupper \& Andrews 2007) [53] & & Referral rates, completion rates & Supply \\
\hline (Blum et al. 2012) [55] & $\begin{array}{l}\text { Home-based follow up using semi } \\
\text { quantitative pregnancy test }\end{array}$ & $\begin{array}{l}\text { Sensitivity and specificity of a pregnancy test } \\
\text { and women's usability }\end{array}$ & Supply-demand \\
\hline \multirow[t]{2}{*}{ (Cameron et al. 2012) [42] } & $\begin{array}{l}\text { Home-based follow up using nurse-led } \\
\text { telephone calls }\end{array}$ & $\begin{array}{l}\text { follow-up rates, efficacy for detecting ongoing } \\
\text { pregnancies and women's satisfaction }\end{array}$ & Supply-demand \\
\hline & $\begin{array}{l}\text { In patient/clinic based telemedicine } \\
\text { (MTOP) }\end{array}$ & & \\
\hline $\begin{array}{l}\text { (Grindlay, Lane \& Grossman } \\
\text { 2013) [57] }\end{array}$ & & Women and staff experience and satisfaction & Demand \\
\hline (Grossman et al. 2011) [58] & & $\begin{array}{l}\text { Completion rates, women's satisfaction, } \\
\text { preferences and recommendations }\end{array}$ & Demand \\
\hline \multirow[t]{2}{*}{ (Grossman et al. 2013) [59] } & & Abortion rates, distance women travelled & Supply-demand \\
\hline & Outpatient STOP & & \\
\hline $\begin{array}{l}\text { (Doran \& Hornibrook } \\
\text { 2014) [22] }\end{array}$ & Outpatient STOP & Experience of access to facility & Demand \\
\hline (Hamoda et al. 2005) [47] & MVA under local anaesthesia & Completion rates, satisfaction, anxiety levels & Supply-demand \\
\hline (Pillai et al. 2015) [51] & MVA under local anaesthesia & $\begin{array}{l}\text { Completion rates, Pain, complications, } \\
\text { contraception uptake, cost }\end{array}$ & Supply-demand \\
\hline \multirow[t]{2}{*}{ (Sharma \& Guthrie 2006) [52] } & $\begin{array}{l}\text { MVA under local anaesthesia and } \\
\text { telephone booking service }\end{array}$ & $\begin{array}{l}\text { Number abortion completions, of telephone } \\
\text { consultations, referrals, and staff satisfaction }\end{array}$ & Supply \\
\hline & Abortion care components & & \\
\hline (Lipp 2009) [48] & STOP and MTOP services & Service provision types & Supply \\
\hline $\begin{array}{l}\text { (Oliveras, Larsen \& David } \\
\text { 2005) [63] }\end{array}$ & $\begin{array}{l}\text { MVA STOP, MSTOP, dilation and curettage, } \\
\text { post-abortion family planning counselling }\end{array}$ & Women's satisfaction & Demand \\
\hline \multirow[t]{2}{*}{ (David et al. 2007) [62] } & Post abortion care program & Contraception counselling delivered & Supply \\
\hline & Care delivery program type & & \\
\hline \multirow[t]{2}{*}{$\begin{array}{l}\text { (Graham, Jayadeva \& Guthrie } \\
\text { 2010) [45] }\end{array}$} & Integrated care pathway, MTOP and STOP & $\begin{array}{l}\text { Re-admission rate, contraception advice } \\
\text { received and uptake }\end{array}$ & Supply \\
\hline & Referral, booking and counselling & & \\
\hline $\begin{array}{l}\text { (Dodge, Haider \& Hacker } \\
\text { 2012) [56] }\end{array}$ & Referral advice & Referral rates & Supply \\
\hline (Esen et al. 2006) [43] & Referral process & Waiting times & Supply \\
\hline $\begin{array}{l}\text { (Finnie, Foy \& Mather } \\
\text { 2006) [44] }\end{array}$ & Referral process & $\begin{array}{l}\text { Waiting times within the pathway to induced } \\
\text { abortion, women's rating of care, GPs' attitudes } \\
\text { and self-reported practice }\end{array}$ & Supply-demand \\
\hline (Mason 2005) [49] & Public service referral process & $\begin{array}{l}\text { Length of wait, number of professionals involved, } \\
\text { women's satisfaction }\end{array}$ & Supply-demand \\
\hline \multirow{2}{*}{$\begin{array}{l}\text { (Norman, Hestrin \& Dueck } \\
\text { 2014) [67] }\end{array}$} & Toll free options service provision & Use rate & Supply \\
\hline & Facility type & & \\
\hline $\begin{array}{l}\text { (Gupta \& Kapwepwe } \\
\text { 2007) [46] }\end{array}$ & NGO & Completion rates & Supply \\
\hline (Jones \& Jerman 2013) [60] & Public and private clinics & Distance travelled & Supply \\
\hline
\end{tabular}


Table 5 Approach to abortion service, outcomes, access dimension and perspective (Continued)

\begin{tabular}{|c|c|c|c|}
\hline $\begin{array}{l}\text { (Kimport, Cockrill \& Weitz } \\
\text { 2012) [61] }\end{array}$ & Public and private clinics & Experience of facility structure and process & Demand \\
\hline $\begin{array}{l}\text { (Nickson, Smith \& Shelley } \\
\text { 2006) [23] }\end{array}$ & Private services & $\begin{array}{l}\text { Distance travelled, money and time expended } \\
\text { undertaking travel, and reasons women chose } \\
\text { particular clinics }\end{array}$ & Supply-demand \\
\hline (Silva \& McNeill 2008) [64] & Public and private clinics & $\begin{array}{l}\text { Number of services, return trip driving } \\
\text { distance }\end{array}$ & Supply \\
\hline $\begin{array}{l}\text { (Silva, McNeill \& Ashton } \\
\text { 2011) [65] }\end{array}$ & $\begin{array}{l}\text { Public and private clinics offering } \\
\text { MTOP vs single day services }\end{array}$ & Timeliness & Supply \\
\hline (Snook \& Silva 2013) [66] & Community based services & Safety and timeliness & Supply \\
\hline
\end{tabular}

a clinic close by even if they preferred to do so [57]. Women also stated that this saved them time, the stress of driving to a facility and the need to take time off work. The researchers found that women living more than 80 $\mathrm{km}$ from the closest facility that offered STOP were more likely to obtain an abortion after the introduction of telemedicine $[58,59]$.

Accessibility in the studies reviewed is manly discussed in relation to constraints. The time and travel distance required to access abortion services is examined in a number of studies included in the review with accessibility for rural women and adolescents more disadvantaged according to three papers. The statistical model in Jones et al. US study showed that having an early abortion was not associated with increased travel but that women who lived in rural areas and those who lived in a state with a 24-h waiting period were more likely to experience increased travel [60]. In Australia, Nickson found that there were limited rural services and adolescents were 2.5 times more likely than other women to travel more than $100 \mathrm{~km}$ (18.2\% compared with $7.8 \%$, OR = 2.5, $95 \%$ CI 1.5-4.2, $p<0.001$ ) [23]. Interstate travel in order to access an abortion was noted in two Australian studies $[22,23]$ with public transport largely unavailable [22].

A qualitative study of women's experiences of three abortion clinics in the US noted that women sometimes made a decision to travel a greater distance to specific clinics to avoid protesters and the associated stress [61]. Avoiding protesters was also noted by women as one reason for selecting a particular clinic in an Australian however, most women selected the clinic on where they obtained their abortion on proximity to their place of residence [23].

Several studies were included in the review revealed issues regarding referral and the accessibility of services. Dodge et al. [56] studied referral to abortion services in America from staff at facilities that did not provide abortion showing that less than half of the women received direct referrals. Women who did not prompt the staff for a referral during a call to facilities in States with less restrictive abortion laws were significantly more likely to be provided with direct referrals $(p=0.006)$ than women who called facilities in more restrictive states. Accessibility was also hampered according to women in an Australian study by slow referrals from health providers [22]. In the UK it was found that referral for abortion was mostly from general practitioner doctors (GP) [44]. However, a review of clinic records in one area in the UK shows that about half of the patients were referred without a Certificate "A" being completed by the referring GP, the reasons for this in some cases was due to issues doctors had with abortion and their conscientious objection to TOP. It was suggested that this may impact upon the speed at which women are able to obtain and abortion [43]. Women in NZ who had more than one visit from their referring doctor also experienced a longer delay to receive an abortion than those who had a single visit [65].

Timeliness of referral was also examined in studies in relation to established recommendations from the UK Royal College of Obstetricians and Gynaecologists (RCOG). Finnie et al. found that referral targets were largely achieved with $52 \%$ of women seen within $48 \mathrm{~h}$ of making an appointment [44]. In addition, the majority ( $97 \%$ ) of women had abortion procedures within 14 days of clinic appointments [44]. Mason's study found that twelve percent of the women had to wait longer than the 3 weeks, exceeding the RCOG's recommendations regarding referral [49]. The survey of GPs found they referred girls under 16 years who requested an abortion with a parent or guardian [44]. Fewer (72; $52 \%)$ reported that would refer unaccompanied girls under 16.

Services factors were found to affect the speed at which women were able to gain an abortion in NZ however the rationale for this is not clear. Silva et al. found that women who obtained their abortion from a private clinic had a significantly shorter delay compared to those who attended public clinics. A shorter delay was also experienced by women who received care from facilities that provided MTOP or single day services and for those women with a lower gestational age [65]. Another NZ study also found that women choosing MTOP had a shorter wait than those obtaining a STOP [66]. 


\section{Affordability}

Some of the studies included in this review examined costs from the perspective of the health system, health professionals and the individual women. In one English primary health care trust Tupper et al. found that a new MTOP service contributed towards cost savings in the UK National Health Service (NHS) budget [53]. The authors compare the cost of their services with those of the 'Payment by Results' national tariff of fixed prices that reflect national average prices for hospital procedures. Costs per case in the new service are outlined as $£ 157$ GBP at set up which was estimated to be $£ 85$ GBP within 5 years of operation. This compares favourably to the $£ 498$ 'Payment by Results' per case on for surgical termination and $£ 423$ for medical termination.

A study undertaken in a different English primary health care trust a year earlier noted that the cost of a MTOP or a STOP (day case) ranged from $£ 462$ to $£ 578$ per patient [52]. Sharma et al. study of a pilot local anaesthetic outpatient STOP service found that the cost could be reduced to $£ 366$ per patient. However, if this involved outpatient consultation the cost was $£ 217$ per patient which could be further reduced to $£ 177$ if the nurse telephone clinic was used. The nurse telephone clinic reduced the time needed by the doctor to assess each patient, which increased the number of patients that could be seen per clinic. Recent research in the UK also examining a local anaesthetic outpatient STOP service found that a cost savings was made of approximately $£ 60000$ per year and that the operating theatre use was reduced by one termination list per week [51].

Interviews with American women accessing a MTOP telemedicine clinic [57] explained that they selected the as it saved them money that they would have had to spend on travel. For some women obtaining a STOP in Australia was very expensive, particularly rural women who in one study said they had borrowed money to cover not only the abortion fee but pay for travel, accommodation and additional childcare costs [22].

\section{Adequacy}

The adequacy of abortion services in terms of the organization of clinics and their hours, of operation, cleanliness and administration processes was the subject of interest in three studies in the review. Interviews with US staff and women revealed that the telemedicine MTOP service offered services more frequently and with a wider range of times available to women than clinic based services. Prior to the telemedicine service staff noted that women could only see doctors on a particular day of the week or month that they visited the clinic, whereas telemedicine enabled women to schedule their consultation any day of the week if needed [57]. Abortion clients from a USAID funded Women and Infant Health
Project who were surveyed in three cities in Russia rated level of comfort and hygiene. Ratings of comfort had the greatest variation with $41 \%$ of clients rating comfort as good, $47 \%$ as fair and $11 \%$ as poor. Less than two per cent of women rated their satisfaction with hygiene as poor while $69 \%$ reported satisfaction with hygiene as good, [63]. Interviews with women obtaining abortions from three clinics in the US described women's experiences of antiabortion protesters outside and the clinics' security measures in response [61]. The findings show that both experiences served to increase women's feelings of stigmatisation. Women described being "buzzed in" passing through metal detectors and paying in cash that made the process seem impersonal and illicit.

\section{Acceptability}

Four of the six papers in this review, that describe the acceptability of services that enable women to terminate their pregnancy at home rather than return to the hospital or clinic after a MTOP, report on women's acceptability of this practice. A survey of 100 women in the UK found that women chose to go home as soon as possible after the procedure $(53 \%)$ and to be in the privacy of their own home (47\%). The majority of women did not regard the amount of pain or bleeding to be a considerable issue [41]. These findings concur with another survey of 127 women who reported that they were adequately prepared for the amount of bleeding and pain they experienced at home [50]. Women in both surveys were satisfied with the procedure with most women in Cameron et al. research $(84 \%)$ stating they would recommend home medical abortion [41].

Blum et al. research in the US examined women's acceptability of a test to detect continuing pregnancy at home after their MTOP. The majority of women surveyed found the test easy to use with two thirds ( $58.1 \%$, $n=190)$ correctly identifying the need to return to the clinic based on the home test reading measures of serum human chorionic gonadotropin being the same or higher than their baseline level [55].

When offered, Cameroon et al. [42] found telephone follow up and self-performed pregnancy testing after their MTOP was more popular with women in the UK up to 9 weeks gestation than return visits to the hospital (476 out of 619 women or $77 \%$ ). Women stated that this was more convenient, less stressful and reduced the need for travel. The majority found the follow up calls from nurses re-assuring and would likely recommend this approach to others. Most women also expressed their preference for a low-sensitivity urine pregnancy test at 2 weeks after their MTOP (97\%). However, if given a choice the majority indicated a preference for selfassessment without a follow-up phone call (52\%) while $43 \%$ stated that they would have been 'unlikely' to choose self-assessment. 
In contrast to women in the study by Grossman et. al. [58] women in Grindlay et al. research paper [57] reported that they were not comfortable with the telemedicine approach to MTOP. These women felt it did not enable them to access other reproductive health services at the same time and that they were unsure about the service as it had not been recommended and was unknown by family and friends. Despite this many women who experienced the telemedicine consultation stated that they were satisfied and comfortable with a video communication as it was already part of their daily lives. Women noted that remote service provision reduced stigma and embarrassment and felt less intimidated. However, others felt that they would have preferred having a personal interaction with a provider and were concerned about privacy issues with a webcam. Most staff found telemedicine acceptable to deliver to women noting issues with detecting women's Rhesus (RH) status. which led to delays in accessing the service. National guidelines recommend the administration of anti-D immunoglobulin to Rh (D) negative women within $72 \mathrm{~h}$ of an MTOP in order to prevent maternal sensitisation and an adverse effect on a future pregnancy $[68,69]$. In the Grindlay et al. study, new providers of telemedicine based abortion services lacked the capability for on-site testing resulting in additional communication with women and third parties in order to establish their Rh status prior to the clinic visit.

Three studies included in this review included an examination of women's acceptability of early STOP services in particular MVA under local anaesthesia. One study found women experienced little pain with $79 \%$ giving a pain score of 3 or less out of 10 [51]. Women were found to recover quickly from their anxiety after their abortion with visual analogue scales, indicating a significant fall in anxiety scores following the procedure $(p<0.01)$. [47]. Women were also satisfied with the procedure in both studies. Eighty-six \% of surveyed women said they would recommend it and $80 \%$ said they would have the same method again in the future [47]. Sharma et al. survey [52] found that staff perceived the patients to be satisfied, "reassured" and "relaxed" and that the provision of a local anaesthesia gave women a choice compared with a general anaesthesia. Telephone consultation was popular with patients according to staff as it was carried out at their convenience in their homes, and with staff as it reduced clinic assessment time. Staff also noted that the telephone booking service with women was acceptable to patients who spent less time in the clinic but that it was more difficult to assess a women's emotional state.

Abortion clients from a USAID funded project surveyed in three cities in Russia were largely satisfied with services received from health professionals Information provided to abortion clients about self-care was the most important predictor of overall satisfaction $(\mathrm{OR}=3.55$, $\mathrm{CI}=1.64,7.69)$. How much the client paid for the abortion did not affect satisfaction with respect to any aspect of care [63]. Despite some women having to wait longer than the 3 weeks recommended by the RCOG guidelines, or in a few cases up to 7 weeks, most women said they were satisfied with the service they received [49]. Women in a survey in the Australian State of Victoria cited the reasons for selecting a private clinic to have their abortion including the reputation of the clinic, quality of information received, as well as good staff and service [23].

Rural Australian women described dissatisfaction with the attitudes of GPs who delayed referral by not providing information concerning options for self-referral. These women stated the need to reduce stigma and negative attitudes to improve access to abortion [22]. American women described the impersonal attitudes of staff and that the denial of support persons for security reasons added to the stigmatisation of the experience. However women's satisfaction was heightened by non-judgemental staff and the presence of a patient advocate [61]. Finnie et al. surveyed women in the UK accessing MTOP and STOP in the UK who overall rated satisfaction, provision of information and staff interaction more highly in secondary than primary care. Women noted that their GPs anti-abortion views were made known to them and although they were referred were provided with very little information concerning their options [44].

\section{Quality}

Quality standards for abortion care were noted in several studies in relation to professional standards, national standards set by the government and local area health standards, as well as women's views of the standard of quality care.

The standards of the RCOG in the UK were referred to in comparison with the results in five studies. In the paper by Essen and colleagues the RCOG minimum referral standard of women seen by a doctor within 2 weeks of referral was found to have been met in $80 \%$ of cases. Of the 340 women in the study 111 patients had their TOP performed within 7 days of their consultation with a doctor in the hospital which was noted as the RCOG ideal standard [43]. The national RCOG standard waiting time of 3 weeks from first appointment with the referring doctor to the procedure was achieved in Finnie et al. study for 56 of 127 women $(44 \%$; $95 \% \mathrm{Cl}, 35-53)$ [44]. In an earlier study the numbers were less with twelve percent of the women having to wait longer than the 3 weeks recommended by the RCOG guideline. In a minority of cases this wait extended up to 7 weeks [49]. Lipp's investigation into services in nine Primary Health Trusts in Wales found that one Trust did not comply 
with referral times of 3 weeks as per clinical guidelines due to service constraints and five of 10 Trusts provided a dedicated assessment clinic as advised for women requesting TOP [48]. Lipp et al. also assessed the quality of written information provided to women prior to abortion in relation to RCOG guidelines that dictate that verbal information should be supported by written impartial, printed material [70]. Five Trusts included written information that was given to women during the initial consultation and on discharge. The information varied in both content and presentation and all material outlined the risks of complications. Tupper et al. [53] note in their evaluation of a new service that the RCOG standards and competences in providing abortion services were observed but no detail of this is provided. One NZ study compared their results of MTOP complications with the standards set by RANZCOG. Complication rates were very reported to be low and within the RANZCOG standards [66].

National standards were cited in a study from Scotland in the UK. The authors audit of facility data noted that National Health Service (NHS) Quality Improvement Scotland standards for sexual health services, which recommend that at least $60 \%$ of women leave an abortion service with an effective method of contraception, was achieved [54].

Graham et al. [45] investigated the use of an integrated care pathway (ICP) in abortion care as a way of incorporating local and national guidelines into everyday practice and managing clinical risk while meeting the requirements of clinical governance in two hospitals in the UK. The researchers undertook an audit of facility data and found that in this context clinical records are a useful tool for high quality record-keeping and to ensure that all women receive the same standard of pre-assessment care. The integrated care pathway (ICP) approach to the provision of quality of MTOP and STOP was developed for two hospitals in the UK and involved a checklist that included STI screening, treatment and partner notification, prophylactic antibiotics at time of abortion, provision of contraception counselling and supply. The checklist also includes readmission investigations such as temperature taking, full blood counts and ultra sound. The ICP evaluation showed that $97 \%$ of women had chlamydia screening before the abortion; all women had a contraceptive discussion and $43 \%$ left using a long-acting reversible method of contraception [45].

\section{Discussion}

This review has identified the dimensions of access to surgical and medical abortion from, clinic or hospitaloutpatient based abortion services, as well as new service delivery approaches utilising a remote telemedicine approach. A range of factors, mostly from studies in the UK and US were found to facilitate improved access to abortion. In particular, flexible service delivery approaches that provide women with cost effective options and technology based services. These could provide possibilities for the Australian health system. However, it is not clear how many services in Australia provide a choice of MTOP or STOP with the situation largely dependent upon the will of private services.

Services that provided women with the choice of either MTOP or STOP [53] and the establishment of nurse-led telephone consultation and outpatient services for STOP $[51,52]$ in this review were found to be cost effective, in demand and the uptake high. Home based MTOP with pregnancy testing and telephone follow up was also reported to be acceptable to women [41, 42, 50, 55]. MTOP delivered by telemedicine was reported to have improved the availability and timeliness of abortion most women found it acceptable, reduced costs to the women and reduced the stress associated with the procedure [57-59]. A private company called the Tabbot Foundation in Australia is trialling the use of video and skype consultations for MTOP that reduces the need for a health services visit except for required tests, or in the case of complications. All of the 303 women involved in the trial at the end of 2015, gave the service a very high rating [71]. MTOP based telemedicine may, as in the American studies in this review, increase the availability of MTOP in Australia, including for women living in rural and remote settings. This could reduce costs associated with long distance travel and enable a timelier delivery of MTOP. In Australia, unlike in the American context, a face to face clinic visit for telemedicine MTOP is not a requirement but and women still need to obtain a blood test to check their rhesus status and an anti-D immunoglobulin injection if Rh negative [72].

The review identified a number of standards, recommendations and targets that provided services and providers with guidance regarding the quality of abortion care including the UK Department of Health targets [53] and RCOG recommendations regarding referral and waiting times [43, 44, 49]. The papers in this review also noted provider competences outlined by RCOG [53] which include the requirement that women leave an abortion service with appropriate information and an effective method of contraception [54]. These standards may provide RANZCOG and the Royal College of General Practitioners with insights to improve practitioner competence in addition to the standards associated with the rate of complications noted in one paper. Targets to reduce waiting times could also be applied nationally in Australia, as well as at State and Territory level, however this would be difficult due to the variability of current service provision. Targets could be applied to public provision but such services may not be apparent and available at all 
public facilities. Assessing progress on targets would require comprehensive and coordinated data collection that does not currently exist across Australia. The collection of abortion data should be alongside other reproductive health indicators and service evaluation. One such approach that might offer guidance are the input, process, outcome and impact indicators recommended by experts at an International workshop on reproductive health indicators and database development [73].

Difficulties accessing abortion services were noted for particular populations in this review, in particular for adolescents and women with limited financial means $[22,23,60,66]$. Little is known about access to abortion services for adolescents who face issues of confidentiality and often negative attitudes of providers [74]. In Western Australia adolescents have been found to have the highest abortion rates [75] which is likely to reflect rates in other States and territories, in line with global figures [76]. Research is therefore needed to ensure that the needs of the highest users of such services are best addressed.

Although not mentioned by the research in this review, other studies have noted higher rates of abortions among migrant women in HIC [77] who face barriers such as poor access to information about contraception, difficulty paying for services, a history of trauma and abuse, and fear of deportation [78]. Sexual and reproductive ill health disproportionately affects migrant and refugee women in Australia and hence is an area of high need requiring attention [79]. In Australia, significant barriers have been documented regarding the access of refugees and migrants to primary health care [80] and recommendations for improving access to general practice have been put forward however, these do not include reproductive health issues such as abortion [81]. Migrants and refugees may also have different expectations of abortion based upon their experiences in their countries of origin. Access for refugees in detention is even more challenging as demonstrated by a recent case of a Somali asylum seeker detained by the Australian Government on the Island of Naru [75].

In our review the descriptions of services were largely public in the UK, NZ, Canada and Russia and private in US and Australia which reflects current service-delivery models in these countries. The model of a public private partnership only appeared in one UK-based study [46]. This difference dictated the papers focus on affordability. Where the cost was born by the state the discussion focused on health systems savings and efficiency, while costs on an individual level featured in the American and Australian papers where women largely access services through private clinics. We found little information concerning ways to best support women from lower socio economic backgrounds with payment if services were unavailable in the public system. While in Australia the universal health system Medicare reimburses costs for STOP and MTOP services, the lack of access through the public system in most states and territories means that women need to access private services leading to an out of pocket gap payment, which can be prohibitive for some women.

Apart from location of services, often concentrated in urban areas, availability is also determined by availability of trained personnel. Staff shortages were noted in papers in our review [22, 53] in the UK and Australia, as well as low knowledge about abortion [44]. Gynaecology and general practice training pathways are variable in some countries [82] and may not include abortion provision. Therefore gynaecologists and GPs must seek additional training to become STOP or MTOP providers, either in their own practices, or within private clinics. In Australia, despite the availability of training for GPs to provide MTOP in their own practices, the uptake of this training and translation into service delivery appears to be relatively low [83]. In the US leadership, the commitment of medical faculties, and the support of professional bodies have been cited as essential components of routine abortion training to increase service provision [84]. This highlights the leadership role of Australian medical, nursing, midwifery and pharmacy faculties and associated professional associations in undertaking to increase provision in public facilities. RANZCOG has developed a training program for its fellows and associates and produced a comprehensive resource for health professionals covering both STOP and MTOP procedures [85]. Task shifting with provision of STOP and MTOP services by, for example registered nurses, is also acceptable to women as noted in this review [52] and is not associated with a higher risk of complications. In Australia task shifting is in its naissance with nurses involved in supporting abortion care for women, however there have been no evaluations of such services. This is in contrast to middle and low income countries where nurses and other mid-level cadres have been found to perform MVA as safely and effectively as doctors in India and Nepal [86, 87].

Studies in the review noted that the anti-abortion views of doctors had slowed the referral process and that GPs made their views known to women [22, 43, 65]. Addressing conscientious objection and, more broadly, provision of sensitivity training for personnel linked to service delivery or referral pathways is critical. Practical training as well as sensitivity training is vital for all levels of service provider including gynaecologists, GPs and other primary care providers, pharmacists and ancillary staff. Our review did not provide insight into how such training should be undertaken and its impact upon access to services. However, doctors have been found to value teaching about the social issues surrounding abortion, as well as clinical ones [82]. Suggestions have been 
made by Kaposy in Canada to allow doctors conscientious objection while also ensuring access. This includes clear advertisement of objection at services and regulation by professional associations to prevent women experiencing referral delays, the refusal of information, or deliberate misinformation [88].

Legal restrictions in different Australian and US states were noted to affect referral and travel in two studies in the review [22, 56]. Variations in the legal status of abortion can also create confusion for providers and women and may present unnecessary barriers to service provision. While there are no barriers to the provision of abortion in public hospitals in Australia when performed within the state or territory-based legal framework, with the exception of South Australia, they appear to be rarely performed in this setting [89]. The lack of abortion services may relate to workforce issues and a lack of leadership, as well as the values of faith based hospital services. While most public hospitals will provide abortions, in certain circumstances hospital policy may impose gestational limits and there is generally no transparent central booking system for women or their GPs to access their abortion services. Kaposy highlights approaches Canada has taken to ensure public access that may be transferrable to Australia states and territories. These measures include national decriminalization and the designation of 33 public hospitals as abortion providers by the government of British Columbia [88].

Only one study in the review specifically examined an integrated care pathway [45]. Comprehensive abortion care incorporates the management of complications, provision of STI testing and treatment, as well as provision of contraception. The integration of abortion services within family planning services and/or sexual health services or conversely integration of family planning/sexual health services within abortion services is important in relation to reducing repeat abortions [90]. Experiences of integrating comprehensive abortion care into health services in HICs are difficult to locate in the literature despite many services, such as those in Scotland, in existence [91, 92]. Studies from LMIC show that it is feasible $[93,94]$ which may provide lessons for wealthier countries.

\section{Conclusions}

Clear ways forward for abortion service delivery in Australia include the provision of choice and flexible options for women, in conjunction with appropriate use of technology and the application of standards to ensure universal access and quality abortion services for all women. The paucity of evidence concerning access to abortion services in Australia, particularly the dimension of availability, adequacy and quality, shows that more research is needed in this area. Of note is a lack of evidence about demand for abortion services including the needs of vulnerable groups of women whose difficulties accessing services may misleadingly indicate a lower demand. Further research is required to examine this perspective. It is imperative that we draw on the success stories of models which minimise unnecessary barriers to women including the necessity to return to clinic for a postMTOP pregnancy test. However, to implement such directives leadership and advocacy is required from Australian medical, nursing, midwifery and pharmacy faculties and their associated professional associations. In addition, political will is needed to decriminalise abortion across all states and territories in Australia and ensure dedicated public provision that is based on comprehensive models tailored for all populations.

\section{Abbreviations}

GP: General practitioner; HIC: High income countries; ICP: Integrated care pathway; LMIC: Low and middle income countries; MTOP: Medical termination of pregnancy; MVA: Manual vacuum aspiration; NZ: New Zealand; RANZCOG: Royal Australian and New Zealand College of Obstetricians and Gynaecologists; RCOG: Royal College of obstetricians and gynaecologists; STI: Sexually transmitted disease; STOP: Surgical termination of pregnancy; UK: United Kingdom; US: United States; USAID: United States of America Aid Agency; WHO: World Health Organization

\section{Acknowledgements}

None.

Funding

This study was unfunded.

Availability of data and materials

All data is available in the public domain.

Authors' contributions

$\mathrm{AD}$ designed the research, led the literature search and analysis and wrote the manuscript. DB contributed to the framework, analysis and discussion. ES and JE contributed to the writing of the background, discussion and edited the paper. All authors read and approved the final manuscript.

\section{Competing interests}

The authors declare that they have no competing interests.

\section{Consent for publication}

Not applicable.

Ethics approval and consent

Not applicable, all data is in the public domain.

\section{Author details \\ ${ }^{1}$ Faculty of Health, University of Technology, Sydney (UTS), P.O. Box 123, Ultimo, NSW 2007, Sydney, NSW, Australia. ${ }^{2}$ Discipline, Gynaecology and Neonatology, University of Sydney, Family Planning New South Wales, 28-336 Liverpool Road, Ashfield, NSW 2131, Australia. ${ }^{3}$ Family Planning New South Wales, 28-336 Liverpool Road, Ashfield, NSW 2131, Australia. ${ }^{4}$ Public Health, Faculty of Health, University of Technology, Sydney (UTS), Jones Street, Sydney, NSW, Australia.}

Received: 8 June 2016 Accepted: 12 October 2016

Published online: 22 October 2016

\section{References}

1. Katz VL. Spontaneous and Recurrent Abortion-Etiology, Diagnosis, Treatment. In: Katz VL, Lentz GM, Lobo RA, Gershenson D, editors. Comprehensive Gynecology. 5th ed. Philidelphia: Elsevier Mosby; 2007. 
2. WHO. Unsafe abortion: global and regional estimates of incidence of unsafe abortion and associated mortality in 2008. Geneva: World Health Organization; 2011.

3. Barot S. Sexual and Reproductive Health and Rights Are Key to Global Development: The Case for Ramping Up Investment. Guttmacher Policy Rev. 2015;18:1.

4. WHO. Safe abortion: technical and policy guidance for health systems. 2nd ed. Geneva: World Health Organization, Department of Reproductive Health and Research; 2012.

5. Souza JP, Widmer M, Gülmezoglu AM, Lawrie TA, Adejuyigbe EA, Carroli G, Crowther C, Currie SM, Dowswell T, Hofmeyr J. Maternal and perinatal health research priorities beyond 2015: an international survey and prioritization exercise. Reprod Health. 2014;11(1):61.

6. New country classifications by income level [http://blogs.worldbank.org/ opendata/new-country-classifications-2016]

7. Weitz TA, Taylor D, Desai S, Upadhyay UD, Waldman J, Battistelli MF, Drey EA. Safety of Aspiration Abortion Performed by Nurse Practitioners, Certified Nurse Midwives, and Physician Assistants under a California Legal Waiver. Am J Public Health. 2013;103(3):454-61. Accessed 14 Oct 2016.

8. Renner RM, Brahmi D, Kapp N. Who can provide effective and safe termination of pregnancy care? A systematic review*. BJOG. 2013;120(1):2331.

9. Ngo TD, Park MH, Shakur H, Free C. Comparative effectiveness, safety and acceptability of medical abortion at home and in a clinic: a systematic review. World Health Organ Bull World Health Organ. 2011;89(5):360-70.

10. Ferreira ALCG, Lemos A, Figueiroa JN, De Souza Al. Effectiveness of contraceptive counselling of women following an abortion: a systematic review and meta-analysis. Eur J Contracept Reprod Health Care. 2009;14(1): $1-9$.

11. Doran F, Nancarrow S. Barriers and facilitators of access to first-trimester abortion services for women in the developed world: a systematic review. J Fam Plann Reprod Health Care. 2015;41(3):170-80.

12. Maternal Perinatal and Infant Mortality Committee. Maternal, Perinatal and Infant Mortality in South Australia 2011. Adelaide: SA Health, Government of South Australia; 2013.

13. Joyce A, Tran B. Induced Abortions In Western Australia 2006-2009 Report Of The WA Abortion Notification System. Perth: Maternal and Child Health Data Integrity Directorate Performance and Quality Division Department of Health, Western Australia; 2011.

14. Chan A, Sage LC. Estimating Australia's abortion rates 1985-2003. Med J Aust. 2005;182(9):447-52

15. Grayson N, Hargreaves J, Sullivan EA. Use of routinely collected national data sets for reporting on induced abortion in Australia. Sydney: AlHW National Perinatal Statistics Unit; 2005

16. Laws P, Grayson N, Sullivan E. Australia's mothers and babies 2004. Perinatal statistics. Sydney: AlHW National Perinatal Statistics Unit; 2006.

17. De Costa C, Douglas H, Hamblin J, Ramsay P, Shircore M. Abortion law across Australia-A review of nine jurisdictions. Aust N Z J Obstet Gynaecol. 2015;55(2):105-11.

18. Shelley JM, Kavanagh S, Graham M, Mayes C. Use of pregnancy counselling services in Australia 2007-2012. Aust N Z J Public Health. 2015;39(1):77-81.

19. De Crespigny LJ, Wilkinson DJ, Douglas T, Textor M, Savulescu J. Australian attitudes to early and late abortion. Med J Aust. 2010;193(1):9-12.

20. De Costa CM, Russell DB, Carrette M. Views and practices of induced abortion among Australian Fellows and specialist trainees of the Royal Australian and New Zealand College of Obstetricians and Gynaecologists. Med J Aust. 2010;193(1):13-6.

21. Betts K. Attitudes to abortion: Australia and Queensland in the twenty-first century. People and place. 2009;17(3):25.

22. Doran F, Hornibrook J. Rural New South Wales women's access to abortion services: Highlights from an exploratory qualitative study. Aust J Rural Health. 2014;22(3):121-6.

23. Nickson C, Smith AMA, Shelley JM. Travel undertaken by women accessing private Victorian pregnancy termination services. Aust N Z J Public Health. 2006;30(4):329-33.

24. Phillips T, Eltherington J, Costa C, Woods C. Knowledge of abortion law and provision of abortion services amongst tertiary students in Far North Queensland. Aust N Z J Obstet Gynaecol. 2012;52(3):299-301.

25. Ensor T, Cooper S. Overcoming barriers to health service access: influencing the demand side. Health Policy Plan. 2004;19(2):69-79.
26. Gulliford M, Figueroa-Munoz J, Morgan M, Hughes D, Gibson B, Beech R, Hudson M. What does' access to health care'mean? J Health Serv Res Policy. 2002;7(3):186-8.

27. Blankenship KM, Bray SJ, Merson MH. Structural interventions in public health. Aids. 2000;14:S11-21.

28. Obrist B, Iteba N, Lengeler C, Makemba A, Mshana C, Nathan R, Alba S, Dillip A, Hetzel MW, Mayumana I, et al. Access to Health Care in Contexts of Livelihood Insecurity: A Framework for Analysis and Action. PLoS Med. 2007;4(10), e308.

29. Tugwell P, De Savigny D, Hawker G, Robinson V. Applying clinical epidemiological methods to health equity: the equity effectiveness loop. BMJ. 2006:332:358-61.

30. Elo S, Kyngäs H. The qualitative content analysis process. J Adv Nurs. 2008; 62(1):107-15

31. CRD. Systematic Reviews CRD's Guidance for Undertaking Reviews in Health Care. York: Centre for Reviews and Dissemination, University of York; 2009

32. Donabedian A. Basic approaches to assessment: structure, process and outcome. Explorations in quality assessment and monitoring. 1980;1:77-128.

33. RCOG. Best practice in comprehensive abortion care. London: The Royal College of Obstetricians and Gynaecologists; 2015.

34. Comprehensive Abortion Care [http://www.ipas.org/en/What-We-Do/ Comprehensive-Abortion-Care.aspx]

35. Baxter S, Blank L, Guillaume L, Squires H, Payne N. Views of contraceptive service delivery to young people in the UK: a systematic review and thematic synthesis. J Fam Plann Reprod Health Care. 2011;37(2):71-84. Accessed 14 Oct 2016

36. Mwaikambo L, Speizer IS, Schurmann A, Morgan G, Fikree F. What works in family planning interventions: a systematic review. Stud Fam Plan. 2011; 42(2):67-82.

37. Moher D, Liberati A, Tetzlaff J, Altman DG, Group TP. Preferred reporting items for systematic reviews and meta-analyses: the PRISMA statement. PLoS Med. 2009;6.

38. Loeber OE. Motivation and satisfaction with early medical vs. surgical abortion in the Netherlands. Reprod Health Matters. 2010;18(35):145-53.

39. Critical Appraisal Skills Programme (CASP) CASP Checklists [http://www. casp-uk.net/casp-tools-checklists]

40. Pluye $P$, Gagnon M, Griffiths F, Johnson-Lafleur J. A scoring system for appraising mixed methods research, and concomitantly appraising qualitative, quantitative and mixed methods primary studies in Mixed Studies Reviews. Int J Nurs Stud. 2009;46(4):529-46. Accessed 14 Oct 2016.

41. Cameron S, Glasier A, Dewart H, Johnstone A. Women's experiences of the final stage of early medical abortion at home: results of a pilot survey. J Fam Plann Reprod Health Care. 2010;36(4):213-6.

42. Cameron ST, Glasier A, Dewart H, Johnstone A, Burnside A. Telephone follow-up and self-performed urine pregnancy testing after early medical abortion: a service evaluation. Contraception. 2012;86(1):67-73.

43. Esen $U$, Koram K, Doherty E, Orife $\mathrm{S}$, Jones A. Termination of pregnancy in South Tyneside. J Obstet Gynaecol. 2006;26(8):791-4.

44. Finnie S, Foy R, Mather J. The pathway to induced abortion: women's experiences and general practitioner attitudes. J Fam Plann Reprod Health Care. 2006:32(1):15-8.

45. Graham O, Jayadeva P, Guthrie K. The use of an integrated care pathway for evidence-based practice and clinical governance in abortion care. J Obstet Gynaecol. 2010;30(4):397-403.

46. Gupta S, Kapwepwe S. Collaboration with the voluntary sector in setting up an early medical abortion service in the PCT. J Obstet Gynaecol. 2007;27(5): 506-9.

47. Hamoda H, Flett GMM, Ashok PW, Templeton A. Surgical abortion using manual vacuum aspiration under local anaesthesia: a pilot study of feasibility and women's acceptability. J Fam Plann Reprod Health Care. 2005;31(3):185-8.

48. Lipp A. Service provision for women undergoing termination of pregnancy: progress in Wales, UK. J Fam Plann Reprod Health Care. 2009;35(1):15-9.

49. Mason L. Referral to a National Health Service-funded abortion clinic. J Fam Plann Reprod Health Care. 2005;31(2):117-20.

50. McKay RJ, Rutherford L. Women's satisfaction with early home medical abortion with telephone follow-up: A questionnaire-based study in the UK. J Obstet Gynaecol. 2013;33(6):601-4.

51. Pillai M, Welsh V, Sedgeman K, Gazet AC, Staddon J, Carter H. Introduction of a manual vacuum aspiration service: a model of service within a NHS Sexual Health Service. J Fam Plann Reprod Health Care. 2015;41(1):27-32 
52. Sharma S, Guthrie K. Nurse-led telephone consultation and outpatient local anaesthetic abortion: a Pilot Proiect. J Fam Plann Reprod Health Care. 2006; 32(1):19-22.

53. Tupper C, Andrews SS. Setting up an outpatient service for early medical termination. J Fam Plann Reprod Health Care. 2007;33(3):199-202.

54. Astle H, Cameron ST, Johnstone A. Comparison of unscheduled reattendance and contraception at discharge, among women having the final stage of early medical abortion at home and those remaining in hospital. J Fam Plann Reprod Health Care. 2012;38(1):35-40.

55. Blum J, Shochet T, Lynd K, Lichtenberg ES, Fischer D, Arnesen M, Winikoff B, Blumenthal PD. Can at-home semi-quantitative pregnancy tests serve as a replacement for clinical follow-up of medical abortion? A US study Contraception. 2012;86(6):757-62.

56. Dodge LE, Haider S, Hacker MR. Using a simulated patient to assess referral for abortion services in the USA. J Fam Plann Reprod Health Care. 2012; 38(4):246-51.

57. Grindlay K, Lane K, Grossman D. Women's and Providers' Experiences with Medical Abortion Provided Through Telemedicine: A Qualitative Study. Womens Health Issues. 2013;23(2):E117-22.

58. Grossman D, Grindlay K, Buchacker T, Lane K, Blanchard K. Effectiveness and acceptability of medical abortion provided through telemedicine. Obstet Gynecol. 2011;118(2 PART 1):296-303.

59. Grossman DAMD, Grindlay KM, Buchacker TRN, Potter JEP, Schmertmann CPP. Changes in Service Delivery Patterns after Introduction of Telemedicine Provision of Medical Abortion in lowa. Am J Public Health. 2013;103(1):73-8.

60. Jones RK, Jerman J. How Far Did US Women Travel for Abortion Services in 2008? J Womens Health. 2013;22(8):706-13.

61. Kimport K, Cockrill K, Weitz TA. Analyzing the impacts of abortion clinic structures and processes: a qualitative analysis of women's negative experience of abortion clinics. Contraception. 2012;85(2):204-10.

62. David PH, Reichenbach L, Savelieva I, Vartapetova N, Potemkina R. Women's reproductive health needs in Russia: what can we learn from an intervention to improve post-abortion care? Health Policy Plan. 2007;22(2):83-94.

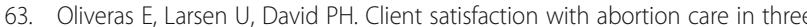
Russian cities. J Biosoc Sci. 2005;37(5):585-601.

64. Silva M, McNeill R. Geographical access to termination of pregnancy services in New Zealand. Aust N Z J Public Health. 2008;32(6):519-21.

65. Silva M, McNeill $R$, Ashton T. Factors affecting delays in first trimester pregnancy termination services in New Zealand. Aust N Z J Public Health. 2011;35(2):140-5.

66. Snook S, Silva M. Abortion services in a high-needs district: a communitybased model of care. J Prim Health Care. 2013:5(2):151-3.

67. Norman W, Hestrin B, Dueck R. Access to Complex Abortion Care Service and Planning Improved through a Toll-Free Telephone Resource Line. Obstet Gynecol Int. 2014;2014:913241

68. RCOG. The use of anti-D immunoglobulin for rhesus D prophylaxis. In: Green-top guideline. London: Royal College of Obstetricians and Gynaecologists; 2011.

69. RANZCOG. Guidelines for the use of Rh (D) Immunoglobulin (Anti-D) in obstetrics in Australia. Melbourne: The Royal Australian and New Zealand College of Obstetricians and Gynaecologists; 2015.

70. RCOG. The Care of Women Requesting Induced Abortio. In: Evidence based Clinical Guideline Number 7. London: Royal College of Obstetricians and Gynaecologists; 2004.

71. Raymond EG, Chong E, Hyland P. Increasing Access to Abortion With Telemedicine. JAMA Intern Med. 2016;176(5):585-6.

72. What's involved the medical termination process [http://www.tabbot.com. au/medical-termination/whats-involved.html]

73. ESCAP. Handbook on Reproductive Health Indicators. New York: Economic and Social Commission for Asia and the Pacific; 2003. Accessed 14 Oct 2016.

74. Davis AR, Beasley AD. Abortion in adolescents: epidemiology, confidentiality, and methods. Curr Opin Obstet Gynecol. 2009;21(5):390-5.

75. Pregnant Somali asylum seeker Abyan had not ruled out abortion, FOl documents reveal [http://www.abc.net.au/news/2016-01-02/pregnantasylum-seeker-wanted-an-abortion-foi-documents-say/7064038]

76. Bearinger LH, Sieving RE, Ferguson J, Sharma V. Global perspectives on the sexual and reproductive health of adolescents: patterns, prevention, and potential. Lancet. 2007;369(9568):1220-31. Accessed 14 Oct 2016.
77. Ostrach B. "Yo No Sabía..."-Immigrant Women's Use of National Health Systems for Reproductive and Abortion Care. J Immigr Minor Health. 2013; 15(2):262-72.

78. Schoevers MA, van den Muijsenbergh ME, Lagro-Janssen AL. Illegal female immigrants in The Netherlands have unmet needs in sexual and reproductive health. J Psychosom Obstet Gynaecol. 2010;31(4):256-64.

79. O'Rourke K. Its time for a new sexual and reprodcutive health strategy for Australia. In: Sexual and Reproductive Health Strategy Reference Group of the Public Health Association of Australia (PHAA), Women's Health Special Interest Group. Canberra: Sexual Health \& Family Planning Association of Australia and the Australian Reproductive Health Alliance; 2008.

80. Lewis V, Marsh G, Hanley F, Macmillan J, Morgain L, Silburn K, Kalucy E, Dwyer J, Rostant J, Mead C. Understanding vulnerability in primary health care: Overcoming barriers to consumer transitions. Canberra: APHCRI, ANU; 2013.

81. Milosevic D, Cheng $\mathrm{H}-\mathrm{H}$, Smith MM. The NSW Refugee Health Service: Improving refugee access to primary care. Aust Fam Physician. 2012;41(3):147.

82. Freedman L, Landy U, Steinauer J. Obstetrician-gynecologist experiences with abortion training: physician insights from a qualitative study. Contraception. 2010;81(6):525-30.

83. Grossman D, Goldstone P. Mifepristone by prescription: a dream in the United States but reality in Australia. Contraception. 2015;92(3):186-9.

84. Guiahi M, Lim S, Westover C, Gold M, Westhoff CL. Enablers of and barriers to abortion training. J Grad Med Educ. 2013;5(2):238-43.

85. RANZCOG. Termination of pregnancy A resource for health professionals. Melbourne: Royal Australian and New Zealand College of Obstetricians and Gynaecologists; 2005.

86. Jejeebhoy SJ, Kalyanwala S, Zavier AF, Kumar R, Mundle S, Tank J, Acharya R, Jha N. Can nurses perform manual vacuum aspiration (MVA) as safely and effectively as physicians? Evidence from India. Contraception. 2011;84(6):615-21

87. Warriner I, Wang D, Huong NM, Thapa K, Tamang A, Shah I, Baird DT, Meirik O. Can midlevel health-care providers administer early medical abortion as safely and effectively as doctors? A randomised controlled equivalence trial in Nepal. Lancet. 2011;377(9772):1155-61.

88. Kaposy C. Improving abortion access in Canada. Health Care Anal. 2010;18(1):17-34.

89. De Costa CM. Medical abortion for Australian women: it's time. Med J Aust. 2005;183(7):378-80.

90. Cohen SA. Repeat abortion, repeat unintended pregnancy, repeated and misguided government policies. Guttmacher Policy Review. 2007;10(2):8-12

91. Lothian Sexual Health [http://www.lothiansexualhealth.scot.nhs.uk/Services/ HowToFindUs/Pages/default.aspx]

92. Sandyford Sexual Health Services [http://www.sandyford.org/]. Accessed 14 Oct 2016.

93. Delvaux T, Sœur S, Rathavy T, Crabbé F, Buvé A. Integration of comprehensive abortion-care services in a Maternal and Child Health clinic in Cambodia. Tropical Med Int Health. 2008;13(8):962-9. Accessed 14 Oct 2016

94. Prata N, Bell S, Gessessew A. Comprehensive abortion care: evidence of improvements in hospital-level indicators in Tigray, Ethiopia. BMJ Open. 2013;3(7), e002873.

\section{Submit your next manuscript to BioMed Central and we will help you at every step:}

- We accept pre-submission inquiries

- Our selector tool helps you to find the most relevant journal

- We provide round the clock customer support

- Convenient online submission

- Thorough peer review

- Inclusion in PubMed and all major indexing services

- Maximum visibility for your research

Submit your manuscript at www.biomedcentral.com/submit 